
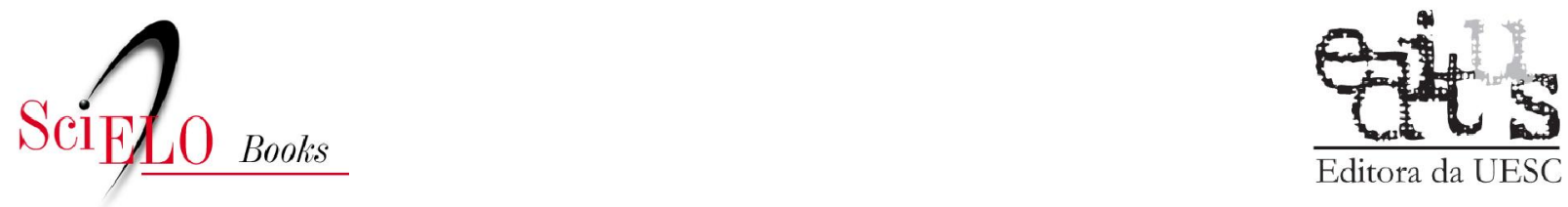

Editora da UESC

\title{
10 - Citogenética e evolução do cariótipo em formigas poneromorfas
}

\author{
Cléa S. F. Mariano \\ Igor S. Santos \\ Janisete Gomes da Silva \\ Marco Antonio Costa \\ Silvia das Graças Pompolo
}

SciELO Books / SciELO Livros / SciELO Libros

MARIANO, CSF., et al. Citogenética e evolução do cariótipo em formigas poneromorfas. In:

DELABIE, JHC., et al., orgs. As formigas poneromorfas do Brasil [online]. Ilhéus, BA: Editus, 2015,

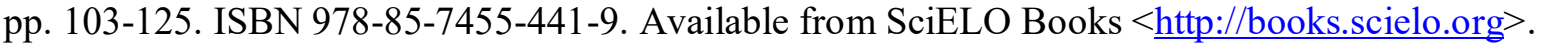

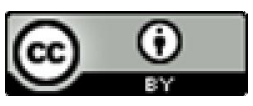

All the contents of this work, except where otherwise noted, is licensed under a Creative Commons Attribution 4.0 International license.

Todo o conteúdo deste trabalho, exceto quando houver ressalva, é publicado sob a licença Creative Commons Atribição 4.0.

Todo el contenido de esta obra, excepto donde se indique lo contrario, está bajo licencia de la licencia Creative Commons Reconocimento 4.0. 


\title{
Citogenética e evolução do
} cariótipo em formigas poneromorfas

\author{
Cléa S.F. Mariano, Igor S. Santos, Janisete Gomes da Silva, \\ Marco Antonio Costa, Silvia das Graças Pompolo
}

\section{Resumo}

A expansão dos estudos citogenéticos a partir do século XIX permitiu que informações acerca do número e composição dos cromossomos fossem aplicadas em estudos evolutivos, taxonômicos e na medicina humana. Em insetos, são conhecidos os cariótipos em diversas ordens onde diversos padrões cariotípicos podem ser observados. Dentre os himenópteros, são conhecidos os números cromossômicos de cerca de 800 morfoespécies de formigas de diversas regiões biogeográficas, embora nem todos esses estudos definam o cariótipo dos organismos estudados. Essas informações correspondem a cerca de 6\% do número de espécies descritas para a família e, mesmo escassas, apontam uma grande variação nos cariótipos conhecidos. Em diferentes subfamílias, padrões distintos de cariótipos são encontrados e, dentre essas, as espécies agrupadas no conjunto artificial denominado "poneromorfas" apresentam interessantes modelos para elaboração de hipóteses acerca da evolução cariotípica que serão apresentados neste capítulo. Dentre as seis subfamílias agregadas sob a denominação poneromorfas, existem informações acerca dos cromossomos de todas as subfamílias e aquela que apresenta mais informações a respeito de cariótipos é também a mais diversa em número de espécies: Ponerinae Lepeletier de Saint Fargeau, 1835. Apenas nessa subfamília observamos cariótipos com número cromossômico variando entre $2 \mathrm{n}=8$ a 120, gêneros com cariótipos estáveis, padrões de cromossomos exclusivos para um gênero como Dinoponera Roger, 1861, variação intraespecífica em espécies de Neoponera Emery, 1901 e a presença de cromossomos com potencial para serem marcadores de uma tribo. As hipóteses aqui lançadas têm por base a análise de mais de 100 cariótipos de poneromorfas. São sucessivamente abordados i) Situação atual do conhecimento citogenético em Formicidae; ii) Conhecimento de citogenética em poneromorfas; iii) Estudos de caso: Tribo Ponerini, possíveis agrupamentos genéricos/cariótipos; iv) Análises dos cariótipos conhecidos para esse agrupamento por meio de modelos como o cariógrafo e uma comparação com outras subfamílias. Esses dados oferecem subsídios para formular hipóteses sobre a evolução dos cariótipos em formigas poneromorfas.

MARIANO, Cléa S.F.; SANTOS, Igor S.; SILVA, Janisete Gomes da; COSTA, Marco Antonio; POMPOLO, Silvia das Graças. Citogenética e evolução do cariótipo em formigas poneromorfas. In: DELABIE, Jacques H. C. et al. As formigas poneromorfas do Brasil. Ilhéus: Editus, 2015. p. 103-125. 
Cytogenetics and karyotype evolution in poneromorph ants - From the 19th century onwards, the continuing development of cytogenetic procedures has allowed information to be gathered that can be applied to studies on evolution and taxonomy. In insects, karyotypes are known for several orders and various karyotype patterns can be observed. Among hymenopterans, chromosome numbers are known for about 800 ant morphospecies from distinct biogeographical regions, although not all of the studies have defined the karyotype of the studied organisms. This karyotype information corresponds to about $6 \%$ of described Formicidae species and, although scarce, has demonstrated that there is large variation among the known karyotypes. Among the different subfamilies, distinct karyotype patterns are found, and of these subfamilies, the species included in the artificial group named poneromorphs are interesting models for the construction of hypotheses regarding karyotype evolution, as discussed in this chapter. For this group, information on chromosomes is available for all six poneromorph subfamilies. The subfamily for which most karyotype information is available, the Ponerinae Lepeletier de Saint Fargeau, 1835, is also the most diverse in species numbers. Only within this family has the following been reported: karyotypes with chromosome numbers ranging from $2 \mathrm{n}=8$ to 120 ; genera with stable karyotypes; chromosome patterns exclusive to a genus such as Dinoponera Roger, 1861; intraspecific variation in species of Neoponera Emery, 1901; and the presence of chromosomes with the potential for being a marker for the tribe Ponerini. The hypotheses advanced in this chapter are based on the analysis of more than 100 karyotypes of poneromorphs. We here discuss: i) the state of the art of cytogenetics of Formicidae; ii) cytogenetics of poneromorphs; iii) case studies concerning the tribe Ponerini, with possible groupings of genera/karyotypes; iv) analysis of known karyotypes for this grouping using methods such as the karyograph; and v) a comparison with other subfamilies. These data provide background for formulating hypotheses on karyotype evolution in poneromorph ants.

\section{Citogenética}

Em comparação com outras disciplinas da biologia, a citogenética pode ser considerada recente, pois somente apareceu no final do século XIX. Os primeiros estudos descreviam os processos de mitose e a presença de cromossomos denominados de "material cromático nuclear" (SUMNER, 2003). Rapidamente foi possível descrever cromossomos mitóticos em animais (FLEMMING, 1882) e estes estudos são considerados os pioneiros da citogenética.

A expansão dos estudos citogenéticos permitiu que esta disciplina fosse aplicada em estudos evolutivos, taxonômicos e na medicina. Em insetos, diversos exemplos podem ser apontados em ordens como Lepidoptera (KANDUL et al., 2007; LUKHTANOV et al., 2011), Diptera (SBILORDO et al, 2010) e Hymenoptera, entre outras (GOKHMAN, 2006). Nessa classe de Hexapoda, diversos modelos cromossômicos foram definidos como, por exemplo, os cromossomos politênicos em dípteros quironomídeos (METZ, 1916).
Na ordem Hymenoptera, os trabalhos de Crozier (1975) e Imai e colaboradores $(1977,1984 a, b)$ foram importantes contribuições ao conhecimento citogenético, os quais determinaram o cariótipo de centenas de espécies de diferentes regiões geográficas cujas informações forneceram a base para a elaboração de uma teoria de evolução cariotípica.

A análise de cromossomos mitóticos é utilizada com frequência em estudos citotaxonômicos em alguns grupos de eucariotos (IMAI, 1983; BAIMAI, 1998; MENEZES et al., 2014). Através do estudo de cariótipos, além do número e morfologia dos cromossomos, é possível inferir sobre diferenciação de espécies, grau de parentesco entre estas, processos evolutivos tais como especiação simpátrica e/ou alopátrica, e posição filogenética dos taxa estudados, assim como elaborar hipóteses sobre a evolução dos cariótipos e seu papel na especiação (LUKHTANOV et al., 2006; LORITE; PALOMEQUE, 2010).

A princípio, cada espécie tem um cariótipo (que é o conjunto cromossômico definido por número + morfologia cromossômica) conservado e 
compartilhado pelos indivíduos, independente da sua distribuição geográfica. Porém, existem mecanismos que causam mudanças nos cariótipos. Estas mudanças podem ser numéricas e/ou estruturais e podem ser observadas em diversos níveis: intrapopulacional (quando mais de um cariótipo é observado em uma única população) (LORITE; PALOMEQUE, 2010); entre populações e entre espécies irmãs ou espécies crípticas (definição de SEIFERT, 2009). Essas alterações são geradas por eventos conhecidos como rearranjos cromossômicos, os quais alteram o número e/ou a morfologia dos cromossomos (Figuras 10.1, 10.2).

As alterações numéricas referem-se à variação no número de cromossomos e são causadas por rearranjos do tipo fissão e fusão cêntricas que podem, respectivamente, aumentar ou diminuir o número de cromossomos (IMAI; MARUYAMA, 1978). Na fissão cêntrica, um cromossomo sofre uma quebra na altura do centrômero e origina dois cromossomos telocêntricos. Na fusão cêntri$\mathrm{ca}$, o inverso acontece: dois cromossomos distintos, acrocêntricos "(cromossomos do tipo A) ou telocêntricos (cromossomos do tipo T), unem-se à altura do centrômero, originando, geralmente, um cromossomo metacêntrico (cromossomos do tipo M)" (SUMNER, 2003).

As alterações estruturais são as inversões e translocações. Inversão é o resultado de duas quebras simultâneas no cromossomo e a reunião (fusão) dos fragmentos em ordenação diferente, sem perda de material genético (IMAI; MARUYAMA, 1978). Este tipo de rearranjo também pode ser considerado, às vezes, como uma alteração no número

Figura 10.1 - Representação esquemática da morfologia dos cromossomos segundo Imai, 1991.

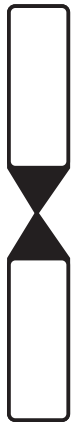

M

Fonte: Original

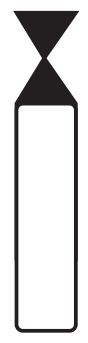

A
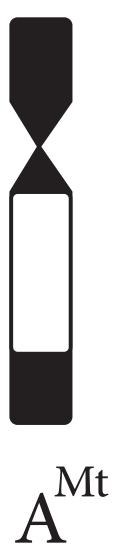

A de braços cromossômicos: este número aumenta quando a inversão é do tipo AM (um cromossomo do tipo A sofre quebras e transforma-se em um cromossomo do tipo M) (Figura 10.2); ou diminui o número de braços quando a inversão é do tipo MA (um cromossomo do tipo M sofre uma quebra na região centromérica e transforma-se em um cromossomo do tipo A) (IMAI; MARUYAMA, 1978). Inversões do tipo MA são consideradas raras em formicídeos porque tenderiam a aumentar a ocorrência de interações entre os cromossomos, provável causa de efeitos deletérios (IMAI et al., 1988). As inversões do tipo paracêntrica (fora da região do centrômero) são imperceptíveis e não são visualizadas por meio das técnicas de caracterização cromossômicas habitualmente utilizadas (IMAI; MARUYAMA, 1978). As inversões não alteram o número de cromossomos em um cariótipo, mas podem ser responsáveis por significativas modificações no genótipo, uma vez que alteram a posição dos genes e, consequentemente, sua expressão.

As translocações envolvem duas ou mais quebras no cromossomo, a remoção de um segmento de um cromossomo e sua junção (fusão) a outro cromossomo, seja esse homólogo ou não (MACGREGOR, 1993) com uma redistribuição do material genético entre os cromossomos. Esse tipo de rearranjo é provavelmente deletério, pois em cariótipos de animais é raramente observado (IMAI et al., 1977).

A poliploidia é o tipo de alteração cariotípica predominante na evolução vegetal (GOULD, 1991) e de alguns grupos de invertebrados (GUERRA, 1988). Em vertebrados, a ocorrência desse
Figura 10.2 - Representação esquemática de rearranjos cromossômicos frequentes em Formicidae. Em a) Fissão cêntrica seguida de deposição de heterocromatina; b) Fusão cêntrica; Inversão pericêntrica.

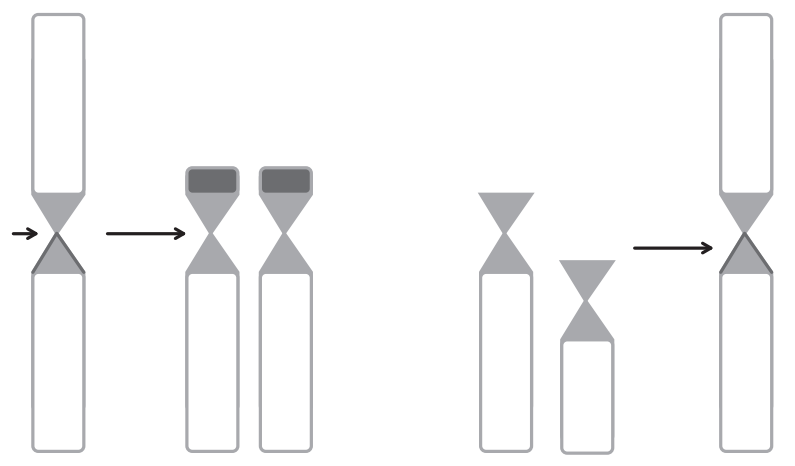

Fonte: Original. 
fenômeno é registrada com menor frequência em grupos como anfíbios (ver, por exemplo, BOGART, 1980). O poliploide pode se originar de um erro meiótico (não-redução cromossômica) ou de uma endomitose em uma célula precursora da meiose (GUERRA, 1988). No entanto, apesar das causas determinantes serem idênticas em qualquer grupo de organismos, os poliploides são mais comuns em alguns grupos do que em outros. Isso se deve a dois fatos: o primeiro é que alguns organismos têm um equilíbrio gênico tão delicado que não toleram dosagens extras de cromossomos e o segundo é que, se o poliploide não apresentar capacidade reprodutiva, ele não será mantido na população e não terá importância evolutiva (GUERRA, 1988). Em formigas, Crozier (1975) e Imai et al. (1977) descrevem casos excepcionais de triploidia (Crematogaster sp. e Vollenhovia sp.) e mesmo tetraploidia (Camponotus sp. e Solenopsis invicta).

A poliploidia apresenta fatores limitantes que lhe são inerentes e pode levar a mudanças nos padrões de metabolismo, nas taxas de desenvolvimento, na regulação de genes e na tolerância fisiológica. Isto pode alterar interações bióticas, tolerância ecológica e comportamento reprodutivo (OTTO; WHITTON, 2000), eventos que podem resultar em especiação, mas que são também deletérios para a espécie. Segundo IMAI et al. (1977), é praticamente impossível a poliploidia em formigas em razão da provável constância do tamanho do genoma em toda a família. Uma síntese sobre o papel dos rearranjos cromossômicos em formigas é discutida por Lorite e Palomeque (2010).

O papel dos rearranjos cromossômicos no processo de especiação é amplamente discutido por diversos autores (WHITE, 1973; IMAI; CROZIER, 1980; KING, 1993; SPIRITO, 1998; IMAI et al., 2001, 2002). Para King (1993), a variação cariotípica pode ser um importante mecanismo de isolamento evolutivo. Em formigas, um aspecto que deve ser levado em consideração para a evolução cromossômica é a haplodiploidia. Esse modelo reprodutivo é consequência da meiose anômala, e a haploidia dos machos, os quais não realizam a meiose tradicional, permite que os formicídeos sejam mais "tolerantes" a rearranjos cromossômicos que outros grupos de organismos (LORITE; PALOMEQUE, 2010).

$\mathrm{Na}$ era da genética molecular, o estudo dos cromossomos permanece importante não apenas porque essas estruturas carregam os genes, mas porque seu comportamento determina os mecanismos de herança (SUMNER, 2003). Além disso, a organização dos cromossomos controla a atividade gênica, uma vez que alterações na sua morfologia provocam modificações na localização dos genes e, consequentemente, sua expressão.

Os cariótipos evoluem gradualmente por meio de múltiplos processos de especiação e/ou variação e cada um desses processos envolve a fixação de um ou poucos rearranjos cromossômicos, quase sempre seguidos de extinção subsequente de espécies ou de variações nos cariótipos intermediários (LUKHTANOV et al., 2011). Esses eventos são comuns na natureza e são possíveis de serem observados em gêneros cujas espécies têm números cromossômicos muito diferentes, os quais são também mais antigos, pois os principais rearranjos que alteram os números cromossômicos são esporádicos (LUKHTANOV et al., 2011). Desse modo, a variação cromossômica exibida por diversos gêneros e até espécies de formigas aqui apresentada pode ser discutida como um evento corriqueiro, de rápida fixação, o que indica um padrão distinto de evolução daquele apresentado por vertebrados, por exemplo (ver LUKHTANOV et al., 2011).

Cerca de 800 morfoespécies de formigas de diversas regiões geográficas têm seus números cromossômicos conhecidos e a maior parte dos estudos citogenéticos diz respeito à constituição dos cromossomos e número cromossômico. Em vista das 12.955 espécies descritas nos Formicidae (AGOSTI; JOHNSON, 2005), consideramos que é escasso o conhecimento acerca da citogenética dessa família. Porém, essas informações, adicionadas àquelas de diversas espécies de outros eucariotos, foram úteis para a elaboração de uma teoria de evolução cariotípica, a Teoria da Interação Mínima (IMAI et al., 1988, IMAI et al , 1994). Esta teoria tem como postulado que "a evolução do cariótipo em eucariotos tende a um aumento no número de cromossomos por meio de processos de fissão cêntrica; estes eventos são essenciais para a não-ocorrência de interações entre os cromossomos, que podem ser facilitadas pelo tamanho longo dos braços cromossômicos" (IMAI et al., 1988). Na evolução do cariótipo, além das fissões cêntricas, são recorrentes rearranjos como inversões pericêntricas, entretanto, há poucos relatos de fusões cêntricas em formigas (IMAI et al., 1986, 1988). Grande parte das análises acerca da evolução do cariótipo em formigas, e também em outros himenópteros, é baseada na interpretação dessa teoria. 


\section{Situação atual do conhecimento citogenético em Formicidae}

O conhecimento citogenético sobre a família Formicidae agrega hoje registros para 14 subfamílias. Uma síntese desse conhecimento é disponível na revisão de Lorite e Palomeque (2010) onde são agrupadas informações para as seis subfamílias alocadas no agrupamento poneromorfas e oriundas de diversas regiões biogeográficas do planeta. Essas informações são, porém, bastante fragmentadas, com poucos gêneros para os quais são conhecidos os cariótipos de mais de cinco espécies.

Diante da escassez das informações e da variação dos cariótipos conhecidos em Formicidae, não é possível apontar uma única direção ou apenas um só evento responsável pela evolução do cariótipo nessa família. Em diferentes subfamílias, padrões distintos de cariótipos são encontrados e, mesmo entre espécies de um mesmo gênero, podem ocorrer variações, sejam numéricas, de morfologia ou de composição cromossômica.

O desmembramento da subfamília Ponerinae originou o agrupamento poneromorfa constituído pelas subfamílias Amblyoponinae, Ectatomminae, Heteroponerinae, Paraponerinae, Ponerinae e Proceratiinae (BOLTON, 2003). Foram descritas até o momento 755 espécies (BOLTON, 2014), a maior parte destas incluídas na subfamília Ponerinae. As espécies destas subfamílias apresentam cariótipos muito variáveis e às vezes exclusivos desse grupo, que serão apresentados neste capítulo.

\section{Metodologia}

As informações acerca dos dados citogenéticos de formigas poneromorfas estão indicadas na Tabela 10.I, que inclui dados inéditos e outros já publicados, com as devidas fontes relacionadas.

As análises aqui apresentadas foram baseadas em número e morfologia cromossômica. Os cromossomos foram classificados, em alguns casos reclassificados, de acordo com a nomenclatura proposta por Imai (1991) de maneira a permitir inferências acerca da direção da evolução do cariótipo em cada táxon baseadas no método cariográfico segundo Imai e Crozier (1980) e Imai et al. (1994) (Figura 10.3).

São apresentadas hipóteses para possíveis direções na evolução do cariótipo em diversos grupos de poneromorfas para os quais temos informações e uma interpretação da evolução do cariótipo baseada na hipótese da variação mediada essencialmente por fissão cêntrica (IMAI; CROZIER, 1980).

\section{Números cromossômicos em poneromorfas}

Estudos citogenéticos já foram realizados em 34 gêneros das seis subfamílias que constituem as poneromorfas, nas quais diversos padrões e até mais de um cariótipo distinto para uma mesma espécie são frequentes. As 182 observações disponíveis de números cromossômicos foram agrupadas e analisadas: na Figura 10.4 é apresentada a frequência do número haploide de cromossomos das espécies que compõem a subfamília Ponerinae, enquanto na Figura 10.5 as informações são relativas às espécies das outras cinco subfamílias que constituem este agrupamento.

Na subfamília Ponerinae, são conhecidos 143 números cromossômicos que apontam a ocorrência de uma grande variação intragenérica ( $n=4-60)$ e uma maior concentração de cariótipos haploides no intervalo $\mathrm{n}=6$-22 (Figura 10.4). De acordo com os dados da Tabela 10.I, é possível observar que apenas o gênero Odontomachus Latreille, 1804 apresenta um "padrão cromossômico", com 10 espécies (de um total de 13) com o número haploide $n=22$. Esse gênero está representado em um dos picos da figura 10.3 $(\mathrm{n}=22)$. O outro pico, representado por $\mathrm{n}=19$, representa espécies dos gêneros Anochetus Mayr, 1861, Ectomomyrmex Mayr, 1867, Hypoponera Santschi, 1938 e Leptogenys Roger, 1861, o que evidencia a heterogeneidade dos cariótipos desta subfamília. Em Ponerinae, a distribuição de números cromossômicos haploides é similar àquela observada em Formicidae por Lorite e Palomeque (2010). Essa diversidade pode ser consequência da posição basal (ou ancestral) desta subfamília (WARD, 2014), uma vez que esses cariótipos representam etapas de um processo evolutivo por meio de sucessivos rearranjos cromossômicos, observados através da composição dos cromossomos de algumas espécies estudadas (SANTOS et al,. 2010; 2012). Os gêneros-irmãos Anochetus e Odontomachus estão entre os mais estudados citogeneticamente, sendo o primeiro o mais diversificado. Apesar de a classificação de Anochetus e Odontomachus como gêneros distintos ser discutível, Santos et al. (2010) concluíram que ambos estão bem delimitados com base nos seus padrões cariotípicos distintos. 
Figura 10.3 - Método cariográfico adaptado de IMAI et al.(1994). Uma vez que o genoma é considerado constante para toda a família Formicidae, um cromossomo de tamanho pequeno corresponde ao aumento no número de cromossomos. Borda 2KA: Limite superior para o número de cromossomos acrocêntricos em células diploides. Borda 2KM: Limite inferior para o número de cromossomos metacêntricos em células diploides. C Fis = Fissão Cêntrica, C Fus = Fusão Cêntrica, P Fis = Fissão Pericêntrica, P Fus =Fusão Pericêntrica, AM inv =Inversão Acrocêntrico-Metacêntrico, MA inv: Inversão Metacêntrico-Acrocêntrico. Fonte: MARIANO et al., 2012.

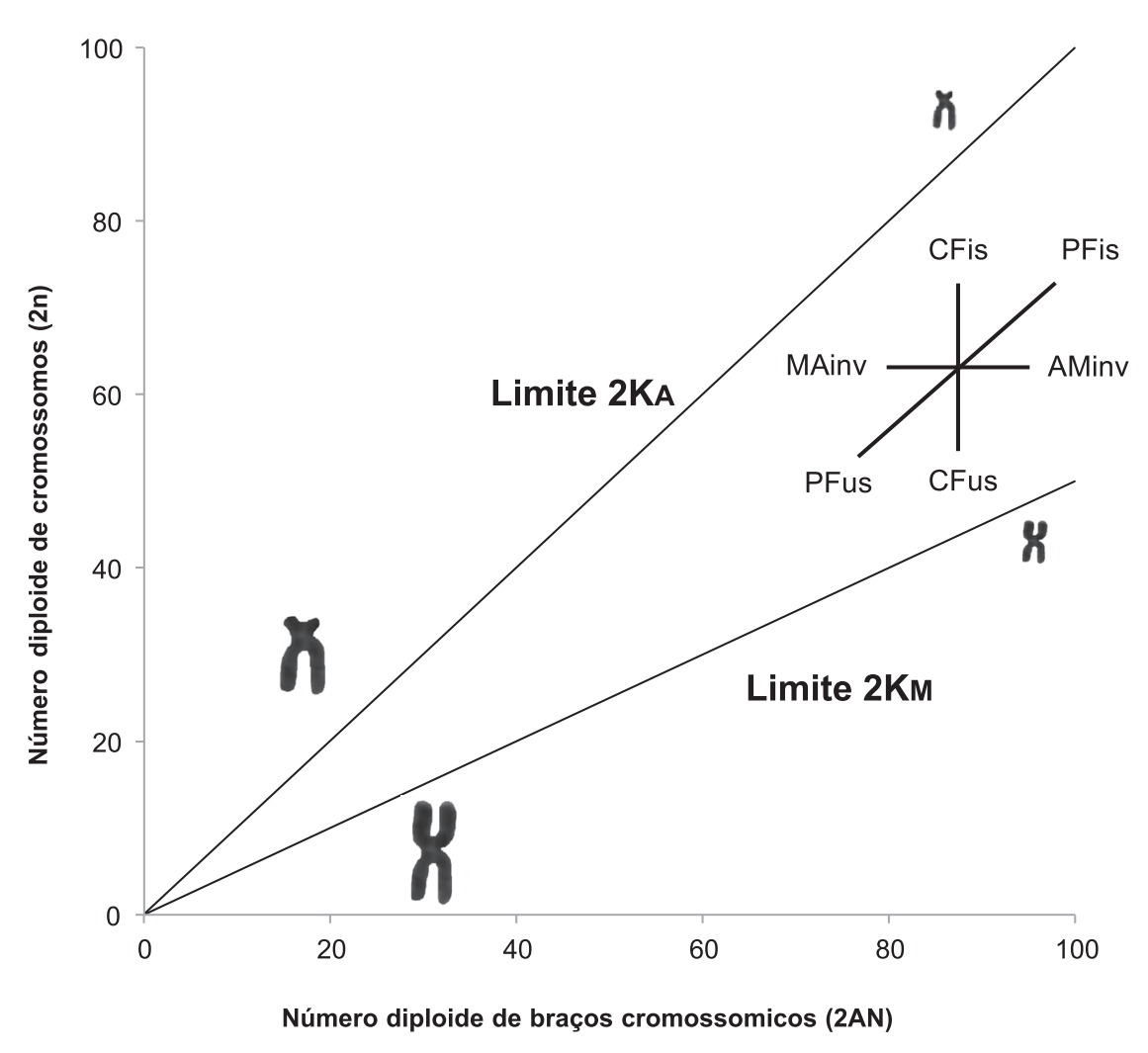

Para as cinco subfamílias que, adicionadas à Ponerinae, compõem o grupo poneromorfas, são conhecidos apenas 39 números cromossômicos. A distribuição dos valores é desigual, uma vez que alguns gêneros são representados por apenas uma espécie e a maior parte das informações representa a subfamília Ectatomminae. Mesmo sendo essa uma amostra menor, também é um espelho da diversidade de números cromossômicos observada em Formicidae (Figura 10.5).

A variação compreende não só o número, mas também a morfologia dos cromossomos. É possível observar cromossomos de morfologia diversa distribuídos nos cariótipos das espécies das seis subfamílias estudadas (Tabela10.I, Figura 10.6).

\section{Estudos de caso}

Vinte e nove espécies da subfamília Ectatomminae têm seus cromossomos conhecidos e estas apresentam uma variação de número diploide de $2 n=16-52$. Existem dados citogenéticos para os quatro gêneros agrupados nesta subfamília e dois destes apresentam variação cromossômica intraespecífica: Rhytidoponera metallica (Smith, 1858) e Typhlomyrmex rogenhoferi Mayr, 1862. Os cariótipos diploides com $2 \mathrm{n}=22-46$ encontrados em $R$. metallica (Tabela 1) são considerados produtos de rearranjos resultantes de eventos de fissão cêntrica, translocação e inversões pericêntricas; dois grupos de cariótipos foram definidos pelos autores, porém operárias de colônias com cariótipos distintos foram consideradas morfologicamente similares. Diante desses resultados, os autores sugerem a ocorrência de dois grupos cariotípicos distintos, nos quais predominam diferentes tipos de rearranjos cromossômicos e apontam a ocorrência de espécies-irmãs (IMAI et al., 1977). Em T. rogenhoferi, os três cariótipos encontrados correspondem a uma clina geográfica na qual os 
Figura 10.4 - Frequência do número haploide de cromossomos das espécies que compõem a subfamília Ponerinae.

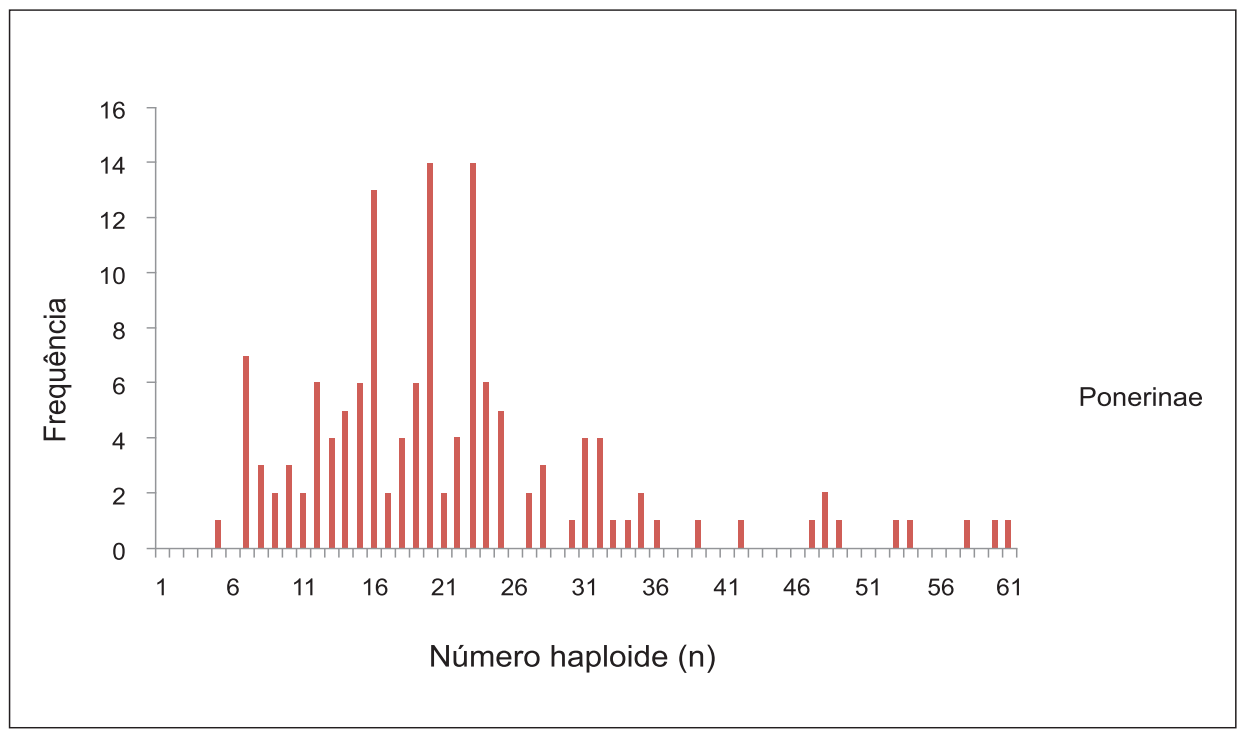

Fonte: Original

Figura 10.5 - Frequência do número haploide de cromossomos das espécies das outras cinco subfamílias de poneromorfas.

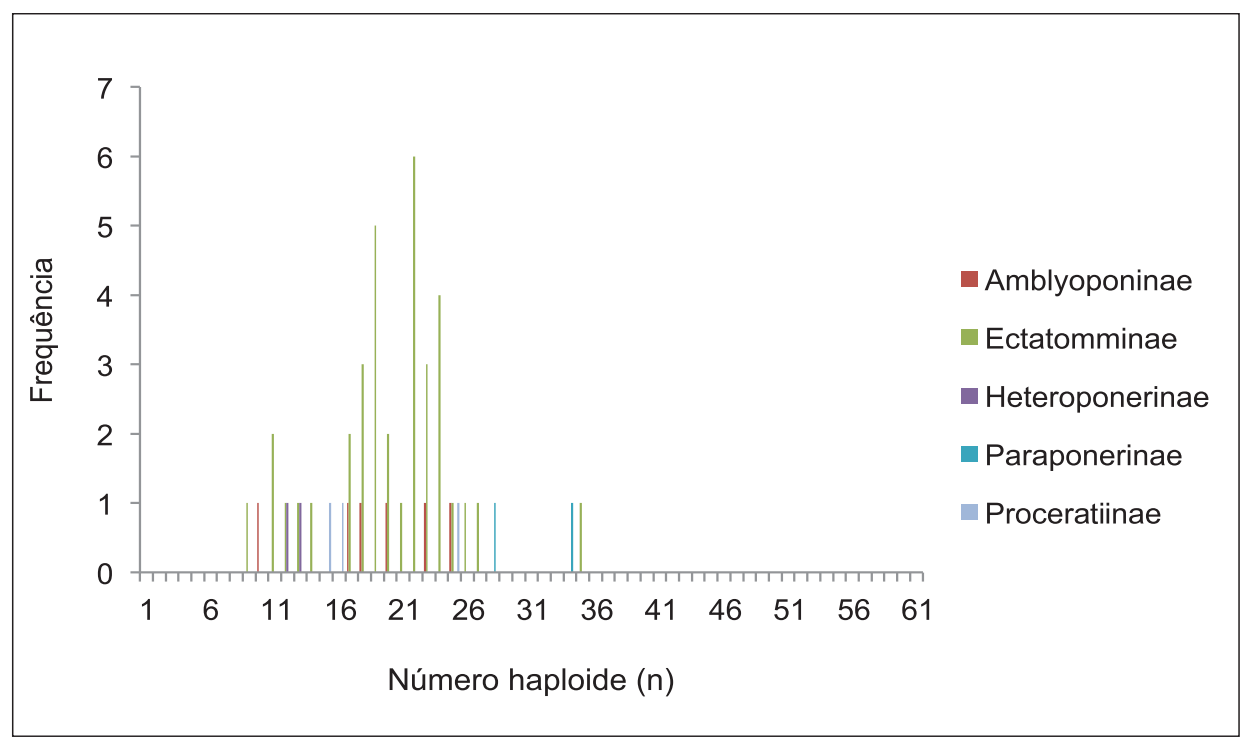

Fonte: Original

pontos de coleta são distantes cerca de $2.600 \mathrm{~km}$ um do outro (Bahia-Pará-Guiana Francesa) com um aumento de um par cromossômico e a ocorrência, nos três cariótipos, de um par do tipo $\mathrm{M}$ assimétrico (MARIANO et al., 2006a). Segundo os autores, a variação observada sugere a ocorrência de eventos de fissão cêntrica como favorecedores da divergência dos cariótipos. Como não foram realizadas investigações complementares com essas populações, como, por exemplo, estudos morfoló- gicos e moleculares, não é possível avaliar o quanto esses cariótipos podem contribuir na diferenciação dessas espécies (MARIANO et al., 2006a).

No cariógrafo (Figura 10.7), os cariótipos de Ectatomminae tendem em direção ao aumento no número de cromossomos por meio de processos de fissão cêntrica, um fenômeno observado para todos os gêneros, especialmente nas espécies de Ectatomma, cuja distribuição de pontos no cariógrafo ilustra claramente a tendência apontada. 
Figura 10.6 - Placas metafásicas de espécies de diferentes subfamílias agrupadas em poneromorfas. Em a) Amblyoponinae - Stigmatomma cleae; b) Ectatomminae Ectatomma permagnum; c) Paraponerinae - Paraponera clavata, d) Heteroponerinae - Heteroponera dolo; e) Ponerinae - Neoponera apicalis;

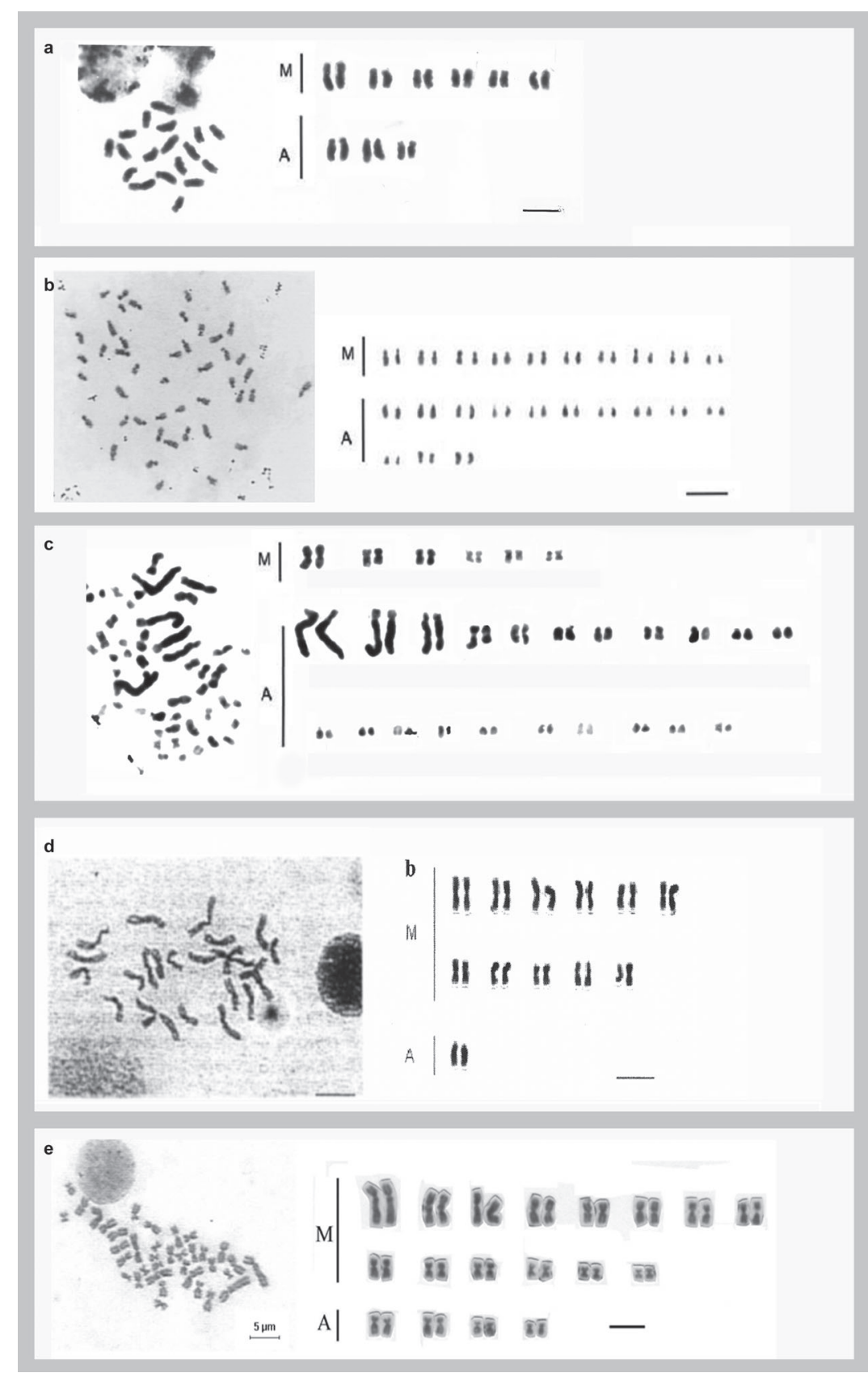

Fonte: a) Original; b) Barros et al. 2008; c) Original; d) Borges et al. 2004; e) Original.

Sobre a composição dos cariótipos, observamos que naqueles compostos por número cromossômico elevado [de acordo com o critério de Imai et al.,1984 ( $\mathrm{n}>11)$ ] a morfologia dos cromossomos é majoritariamente submetacêntrica e acrocêntrica e esses cariótipos originaram-se de processos de rearranjos como fissão cêntrica seguida ou não de inversão pericêntrica (A-M ou $\mathrm{M}-\mathrm{A})$, as quais também podem ser responsáveis ou coadjuvantes em processos de especiação, particularmente nos casos de complexos de espécies crípticas comuns em Ponerinae (nos gêneros Neoponera e Pseudoponera). Cariótipos com elevado número de cromossomos são típicos das espécies de Pachycondyla (P. crassinoda, P. harpax, P. impressa e $P$. striata) e Dinoponera, considerado por Schmidt e Shattuck (2014) gênero irmão de Pachycondyla. 
Figura 10.7 - Cariógrafo de espécies da subfamília Ectatomminae. Os símbolos representam gêneros distintos, indicados na legenda.

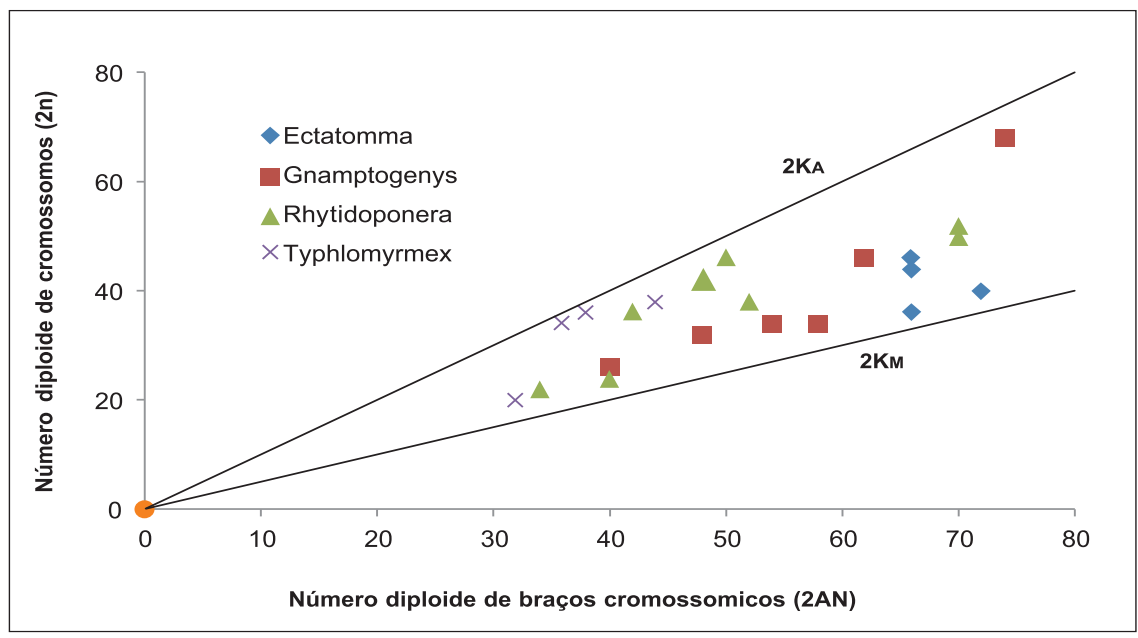

Fonte: Original

Figura 10.8 - Cariógrafo de espécies neotropicais anteriormente alocadas no gênero Pachycondyla. As elipses circulam grupos de espécies mais representativos. O ponto isolado (quadrado) representa o único táxon não incorporado em algum desses grupos.

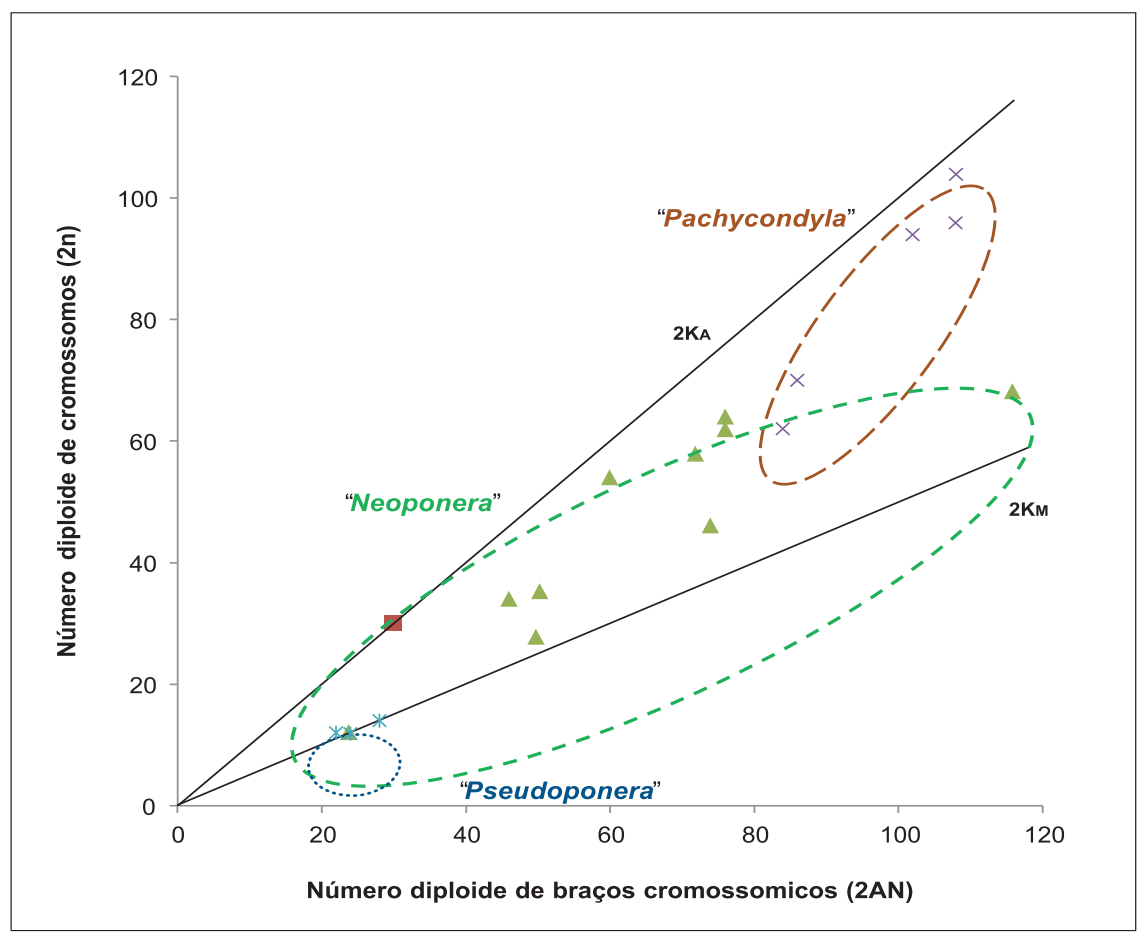

Fonte: Mariano et al., 2012.

A confirmação da polifilia do gênero Pachycondyla (SCHMIDT; SHATTUCK, 2014) foi amparada também por dados citogenéticos (MARIANO et al., 2012), os quais indicavam agrupamentos em espécies neotropicais de Ponerinae alocadas no gênero Pachycondyla (MARIANO et al., 2012; Figura 10.8). A observação dos cariótipos revela similaridade entre as espécies Pachycondyla crassinoda (LATREILLE, 1802), Pachycondyla harpax (FABRICIUS, 1804), Pachycondyla impressa Roger, 1861 e Pachycondyla striata Smith, 1858, as quais se distribuem em um espaço bem definido no cariógrafo, representativo para espécies neotropicais anteriormente alocadas em Pachycondyla (MARIANO et al., 2012; Figura 10.8). 
Placas metafásicas de espécies de Dinoponera e Pachycondyla estão ilustradas na Figura 10.9. Além destas espécies, Bothroponera rubiginosa (EMERY, 1889) e Neoponera metanotalis (LUEDERWALDT, 1918) também possuem cariótipos compostos por elevado número de pequenos cromossomos acrocêntricos (IMAI et al., 1984a MARIANO et al., 2012). Diante da posição filogenética desses dois gêneros (SCHMIDT; SHATTUCK, 2014) seria possível inferir que o número de cromossomos é que determina a composição dos cariótipos, independente do grupo taxonômico. Porém, como apenas uma das 27 espécies de Bothroponera descritas apresenta esse cariótipo e pelo fato dos cariótipos conhecidos das espécies de Neoponera serem muito variáveis (ver discussão adiante), a hipótese do não agrupamento apresentada acima ainda não pode ser comprovada.

Das oito espécies descritas do gênero Dinoponera (LENHART et al., 2013), cinco têm os números cromossômicos conhecidos (SANTOS et al., 2012, Tabela 10.I). Este é o gênero mais próximo de Pachycondyla, assim ambos compartilham características ecológicas, morfológicas e moleculares, além de citogenéticas (SANTOS et al., 2012; SCHMIDT; SHATTUCK, 2014). Além do elevado número cromossômico, estes cariótipos apresentam um par de cromossomos pseudo acrocêntrico incomum em formigas (SANTOS et al., 2012, Figura 10.10). Este par cromossômico é caracterizado pela morfologia particular: os cromossomos possuem tamanho maior que os outros do complemento, com um braço curto heterocromático e um grande bloco heterocromático no final do braço longo, o qual pode ser o resultado de processo de fissão cêntrica seguida de adição de heterocromatina (MARIANO et al., 2008). Análises acerca da composição destes cromossomos em $D$. lucida apontam, na região mediana desses cromossomos, a presença de genes ribossomais confirmados por

Figura 10.9 - Placas metafásicas de espécies de Dinoponera e Pachycondyla. Em a) Dinoponera australis; b) Dinoponera lucida, c) Pachycondyla striata; d) Pachycondyla crassinoda;

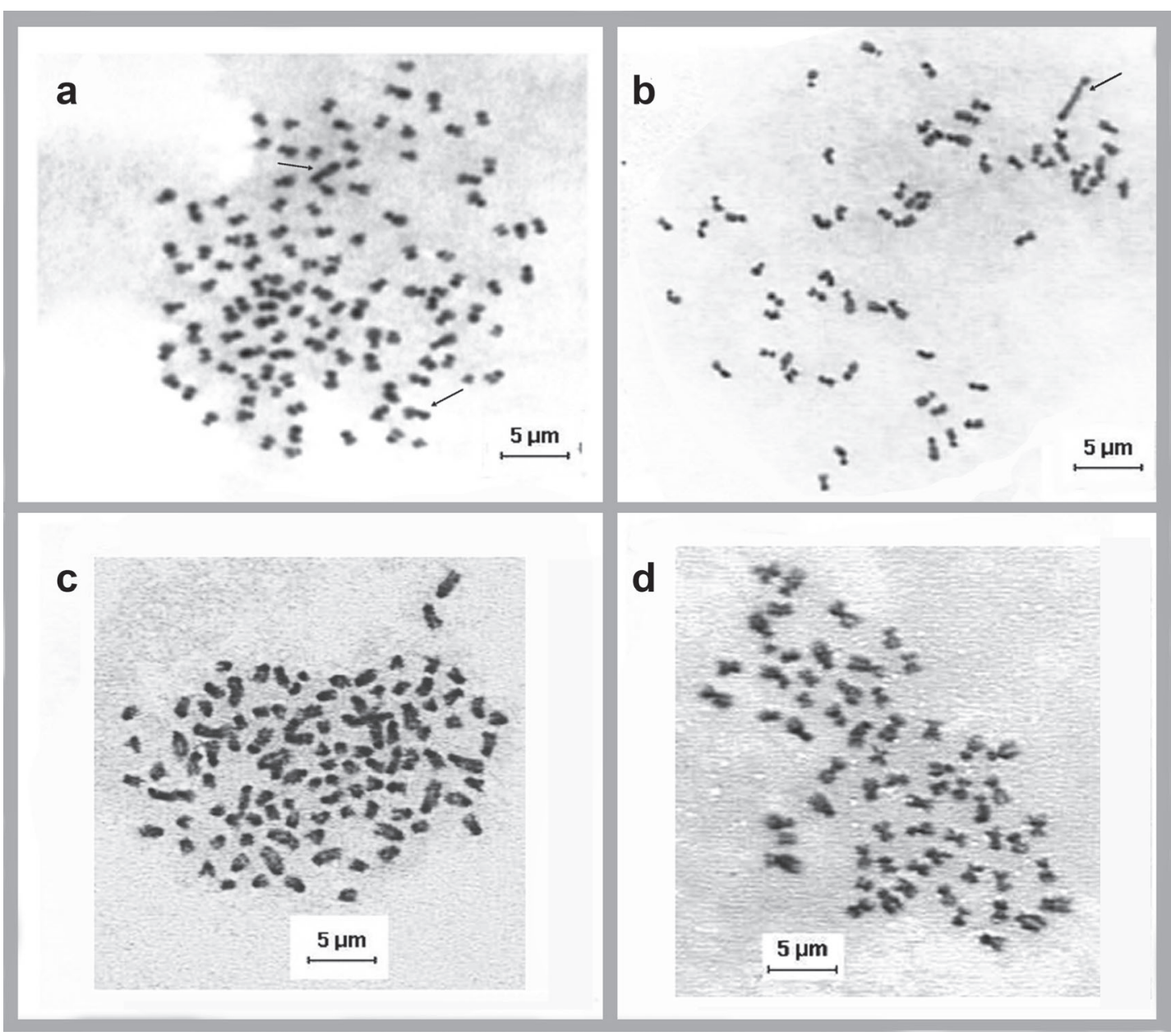

Fonte: a) e b) Santos et al. 2012; c) e d) Mariano et al. 2006b. 
métodos de marcação direta e indireta (MARIANO et al., 2008; BARROS et al., 2009) e indicam que este é um cromossomo promissor a ser utilizado como um marcador citotaxonômico, uma vez que é exclusivo deste gênero.

Com 57 espécies válidas, o gênero Neoponera, de distribuição neotropical, é, dentre os poneríneos, aquele que apresenta espécies com morfologia, ecologia e comportamento dos mais variados (SCHMIDT; SHATTUCK, 2014). A maior parte das espécies tem nidificação arborícola, mas também são encontradas em frutos secos de cacau (SCHMIDT; SHATTUCK, 2014; CASTAÑO-MENESES et al., 2014), cascas de árvores, cavidades de troncos em decomposição no solo e cupinzeiros abandonados (Mariano, dados não publicados). São conhecidos os cariótipos de catorze espécies e para algumas destas espécies ao menos dois cariótipos podem ser observados, uma variação geralmente decorrente da distribuição geográfica da espécie, a qual soma um total de 26 cariótipos para as 14 espécies já estudadas.

As espécies Neoponera apicalis (Latreille, 1802) e Neoponera verenae Forel 1822 compartilham caracteres ecológicos e biológicos bastante parecidos (FRESNEAU, 1994). Não existe nenhum polimorfismo entre as operárias e a morfologia da rainha é bastante semelhante àquela das operárias. Outra característica que elas possuem em comum é a ausência de comportamento de construção que resulta em uma estratégia oportunista de nidificação em cavidades pré-existentes para as quais certamente espécies dos dois conjuntos competem quando vivem em simpatria (DELABIE et al., 1997). Nas matas úmidas com bastante sombra, essas formigas nidificam geralmente em tocos ocos de árvores mortas, onde as condições de umidade e luminosidade são adequadas (DELABIE et al., 2008). Essas espécies representam interessantes questões evolutivas baseadas em dados biogeográficos. A coexistência de duas formas em ambientes terrestres na região Neotropical (WILD, 2005) sugere a ocorrência de dois complexos de espécies crípticas de distribuição alopátrica (DELABIE et al., 2008) com base nas informações citogenéticas (Figura 10.11). Considerando-se N. apicalis e N. verenae como complexos de espécies, estes formam grupos irmãos que provavelmente evoluíram independentemente devido a mecanismos citológicos como fissões cêntricas e inversões pericêntricas documentadas nos cariótipos conhecidos e observadas na análise cariográfica (Figura 10.12). Nesta figura, os cariótipos apresentam uma tendência em direção ao aumento do número cromossômico, porém, por meio de processos distintos: fissões cêntricas seguidas de inversões pericêntricas do tipo AM para $N$. apicalis e fissões cêntricas com poucos processos de inversão para $N$. verenae, espécies cujos cariótipos são compostos, em sua maioria, por cromossomos acrocêntricos.

A tendência observada no gênero Neoponera exemplifica um modelo de evolução que vai ao encontro daquele proposto por Imai et al (1994): os cariótipos sofrem ciclos sucessivos de rearranjos com tendência ao aumento do número cromossômico por fissão cêntrica (Figura 10.13), são bastante heterogêneos, porém alguns agrupamentos são presentes.

\section{Baixo número, grandes cromossomos}

Assim como nos cariótipos compostos por elevados números cromossômicos discutidos acima, naqueles compostos por $\mathrm{n} \leq 11$ a maior parte dos cromossomos é do tipo $M$, são grandes e frequentemente relatados como cariótipos com uma origem recente e passíveis de sofrerem rearranjos do tipo fissão cêntrica (Figura 10.14). Este padrão é encontrado em Amblyoponinae

Figura 10.10 - Representação do par de cromossomos $\mathrm{A}^{\mathrm{MT}}$ presente em espécies do gênero Dinoponera.

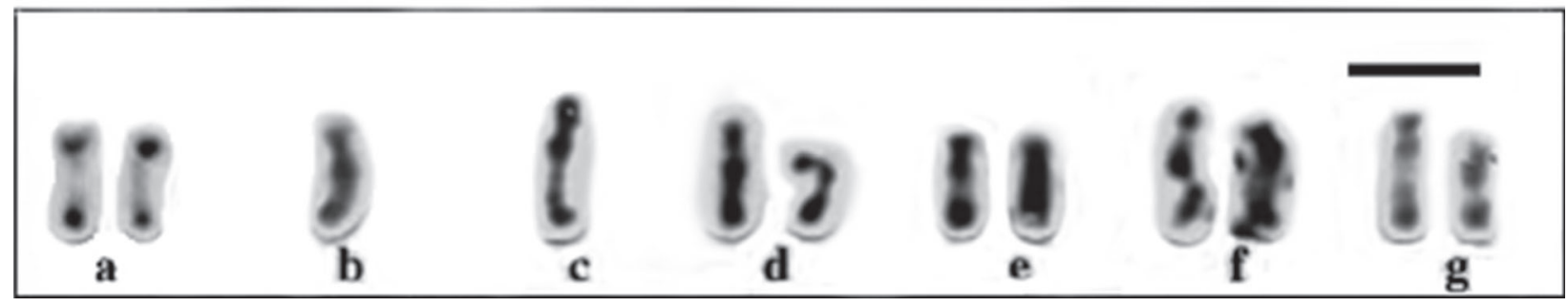

Fonte: Mariano et al., 2008. 
Figura 10.11 - Mapa de distribuição de localidades nas quais é verificada a simpatria entre vários táxons de N. apicalis e N. verenae. Os casos de simetria envolvendo o maior número de espécies estão distribuídos entre as latitudes $5^{0} \mathrm{~N}$ e $10^{\circ} \mathrm{S}$. O diâmetro dos círculos é proporcional ao número de táxons. Círculos cheios: dados da fonte de referência; círculos vazios: dados de Wild (2005).

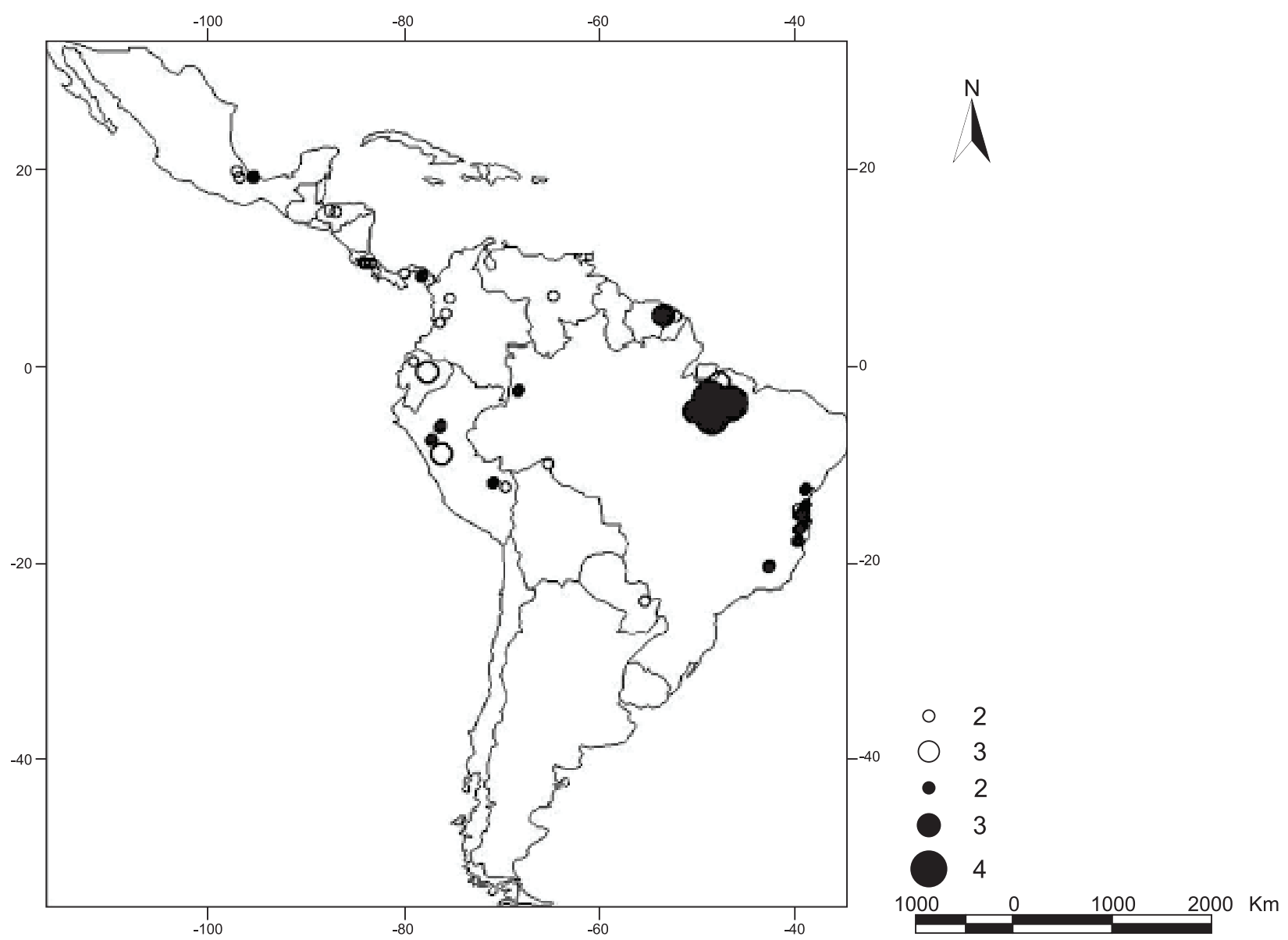

Fonte: Delabie et al., 2008

Figura 10.12 - Cariógrafo de N. apicalis e N. verenae. Os círculos representam os cariótipos de N. apicalis e os losangos os cariótipos de N. verenae.

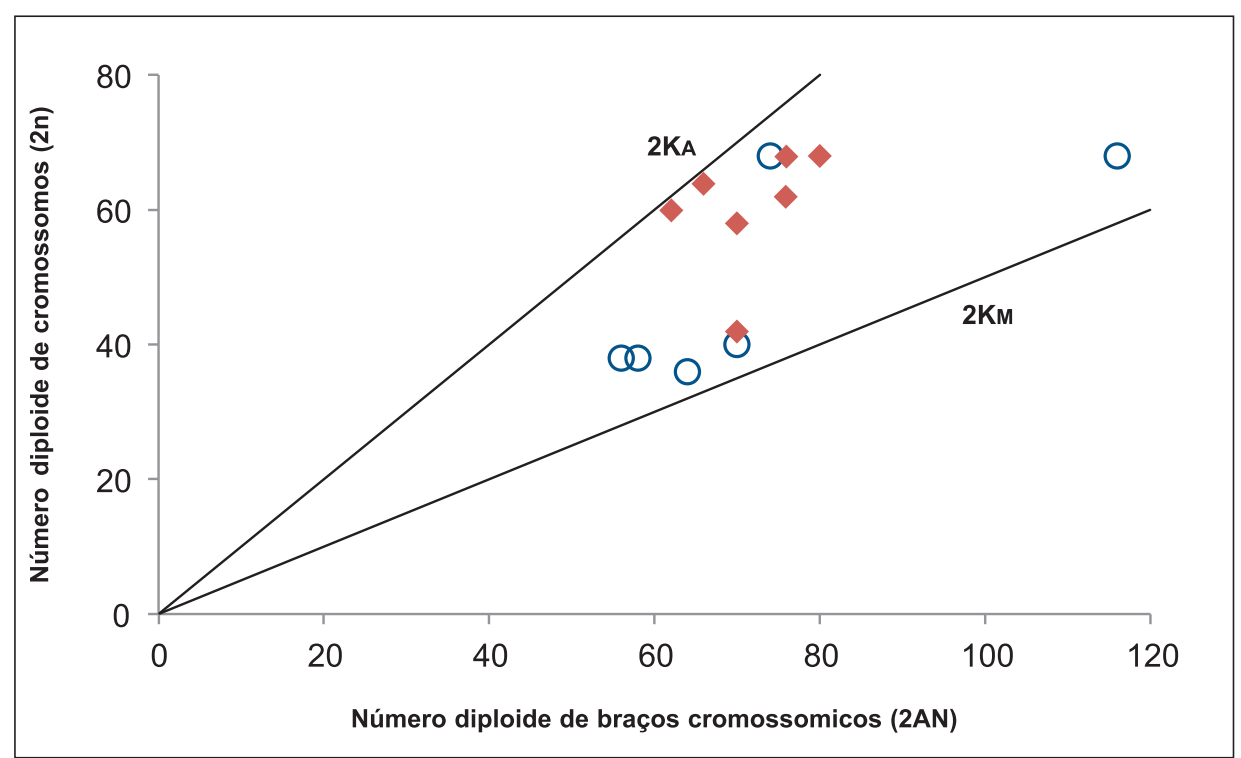

Fonte: Original 
Figura 10.13 - Cariógrafo de agrupamentos de espécies de Neoponera neotropicais. Os círculos elíptcos As elipses circulam os grupos mais representativos; um círculo preto isolado representa uma única espécie, Neoponera marginata que não está incorporada em algum grupo.

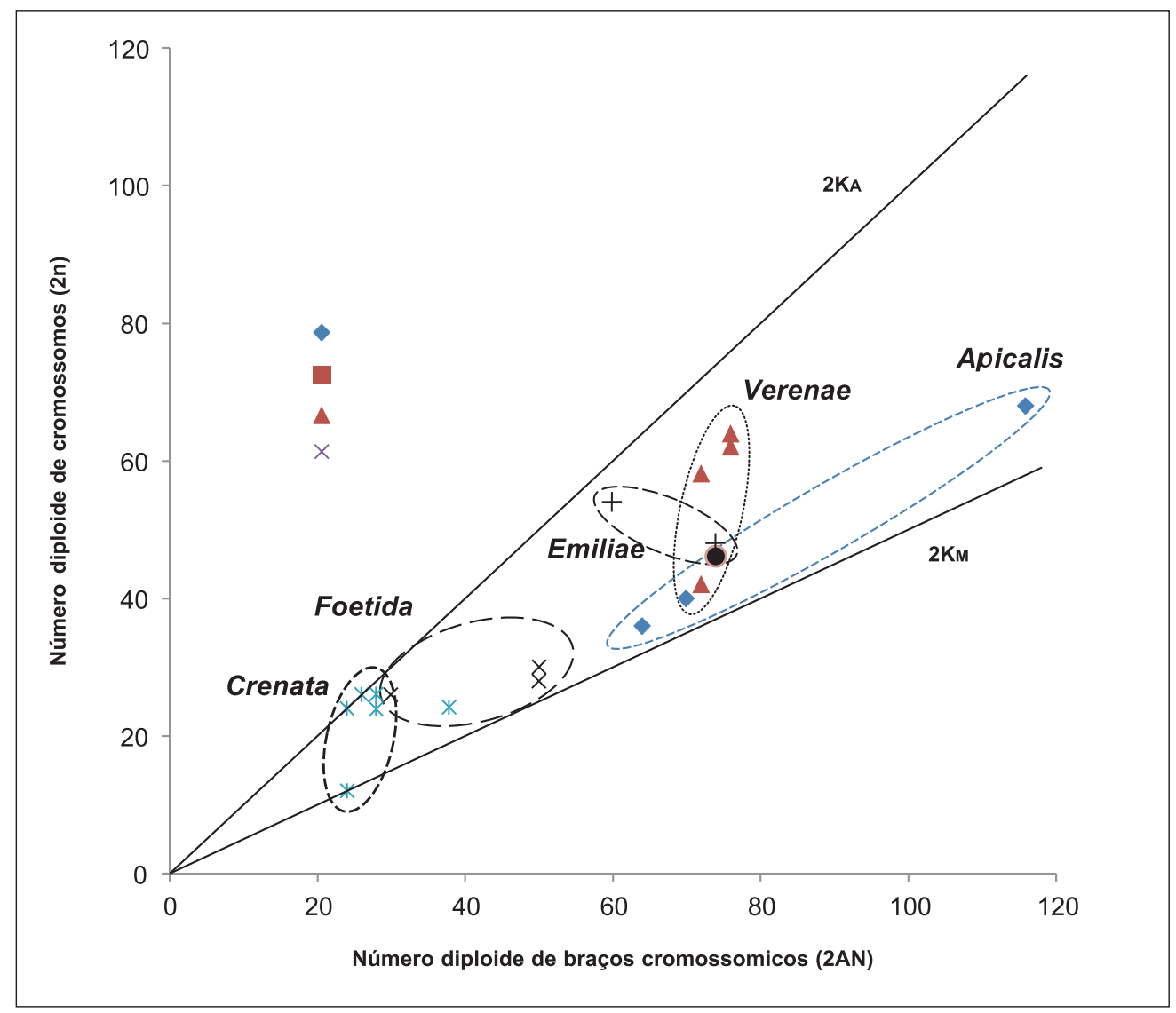

Fonte: Mariano et al., 2012.

(Stigmatomma cleae LACAU; DELABIE, 2002), Ectatomminae (Gnamptogenys spp.,Typhlomyrmex meire LACAU, VILLEMANT; DELABIE, 2004), Heteroponerinae (Heteroponera relicta WHEELER, 1915) e Ponerinae [Pseudoponera gilberti (KEMPF, 1960), Pseudoponera stigma (Fabricius, 1804) e Pseudoponera succedanea (ROGER, 1863); Brachyponera lutea (MAYR, 1862) e Brachyponera luteipes (MAYR, 1862); Cryptopone rotundiceps (EMERY, 1914) e Cryptopone sinensis WANG, 1992; Neoponera unidentata (MAYR, 1862); Ponera scabra WHEELER, 1928 e Thaumatomyrmex sp. 1 (Tabela 10.I).Baixo número cromossômico aparece também nos gêneros Diacamma, Ectomomyrmex e Platythyrea, porém esses cariótipos não são conhecidos.

\section{Considerações finais}

Estes exemplos ilustram a heterogeneidade cariotípica em poneromorfas, sustentam a parafilia do gênero Pachycondyla definida por Schmidt e Shattuck (2014), contribuindo para a compreensão do "problema Pachycondyla" (WARD, 2011) e reforçam a condição ancestral da subfamília Ponerinae, uma vez que grupos distintos seguem padrões evolutivos únicos ( $\mathrm{Fi}$ gura 10.14).

Alguns desses padrões não são exclusivos para as espécies apresentadas aqui, pois são observados em cariótipos de espécies do gênero Myrmecia (IMAI et al., 1994), em um estudo que representa o melhor exemplo da aplicação da citotaxonomia (LORITE; PALOMEQUE, 2010) e reforça a ocorrência de diferentes padrões evolutivos em insetos. Outro exemplo, o estudo de espécies neotropicais anteriormente alocadas em Pachycondyla cujos resultados foram analisados por meio do método cariográfico (Figura 10.8) mostra diversos agrupamentos que dão suporte à parafilia deste gênero: Pachycondyla "stricto sensu" (muitos cromossomos acrocêntricos), Pseudoponera 
Figura 10.14 - Placas metafásicas de espécies c/ $\mathrm{n} \leq 11$. a) Neoponera unidentata; b) Typhlomyrmex meire;c) Stigmatomma cleae; d) Gnamptogenys sp; e) Pseudoponera succedanea;

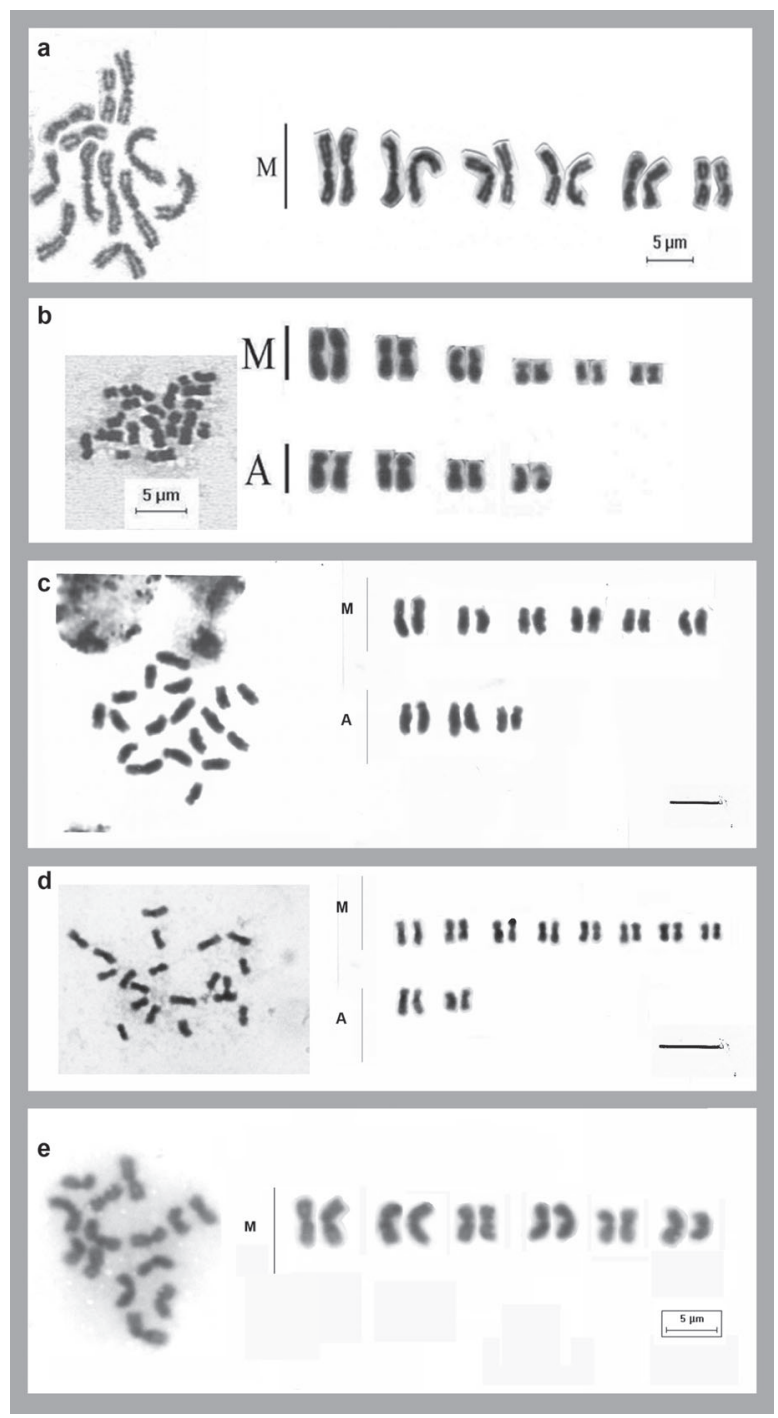

Fonte: a), c), d), e) - imagens originais; b) Mariano et al. 2006a.

(predominância de poucos cromossomos metacêntricos) e a variação encontrada em Neoponera. A distribuição dos pontos sugere que os rearranjos mais frequentes nestes cariótipos foram fissões cêntricas e inversões pericêntricas do tipo A-M e estes rearranjos favorecem um aumento no número cromossômico.

A partir dessas observações podemos verificar a importância da fissão cêntrica como um mecanismo que, ao diminuir a probabilidade de ocorrência de interações entre cromossomos não -homólogos, é um processo favorecido pela seleção (IMAI et al., 1986, 1988, 1994, 2001). Neste cenário, a evolução cromossômica em Formicidae, assim como em Ponerinae, tende ao aumento do número cromossômico, mais que o processo inverso, o de fusão cromossômica.

A diversidade de cariótipos encontrada nas espécies de Ponerinae tem suporte na antiguidade da subfamília e reforça a tendência observada nos cariótipos de Formicidae: o aumento e a diversificação do número cromossômico e da morfologia em uma subfamília basal contrastam com a baixa variação em gêneros de subfamílias mais derivadas como Formicinae e Myrmicinae (WARD, 2014). O mesmo fenômeno de diversidade cariotípicaobservadonasMyrmeciinae australianas (IMAI et al., 1994) leva à ideia muito discutida da condição basal dessas duas subfamílias que não encontra suporte nas filogenias moleculares e morfológicas recentes (MOREAU et al., 2006; WARD, 2014). Em formigas, é reconhecida a ocorrência de complexos de espécies crípticas e espécies-irmãs, espécies morfologicamente pouco distintas com divergência recente (espéciesirmãs) ou que mantêm caracteres convergentes e que não são diferenciadas por meio dos métodos tradicionais de identificação (espécies crípticas) (BICKFORD et al., 2006; SEIFERT, 2009). Nessas espécies, caracteres como o comportamento, a assinatura química e a composição do cariótipo, por exemplo, atuam como mecanismos de isolamento reprodutivo (exemplos em LUCAS et al., 2002). Como este fenômeno tem sido relatado para formigas e outros organismos (LUKHTANOV et al., 2011 por exemplo) é provável que, assim como os conceitos de espécie, múltiplos processos de especiação ocorram constantemente (MAYR, 2001). Nesse contexto, a aplicação de diversos critérios de reconhecimento e descrição de espécies é justificável e estes critérios são testados usando uma abordagem integrativa, a qual consiste na aplicação de estudos complementares como genética molecular, ecologia, comportamento, química e citogenética, entre outras disciplinas (SCHLICK-STEINER et al., 2010), um consenso entre vários autores (PADIAL; LA RIVA, 2010; CRISTIANO et al., 2013). Uma vez que a validade das espécies é reconhecida por outros métodos, a citogenética pode contribuir para o estudo da definição da origem e limite das espécies, contribuindo para a classificação de grupos de formigas cuja delimitação ainda não é bem estabelecida e também para a compreensão das relações de parentesco entre as formigas poneromorfas. 
TABELA 10.I - Dados citogenéticos de poneromorfas; as informações publicadas estão referenciadas na coluna FONTE

\begin{tabular}{|c|c|c|c|c|c|}
\hline Subfamília/Espécie & $2 n$ & $\mathbf{n}$ & Cariótipo diploide & Origem & FONTE \\
\hline \multicolumn{6}{|c|}{ Amblyoponinae } \\
\hline Amblyopone cf. fortis & 44 & 22 & $20 A+24 M$ & Austrália & 1 \\
\hline Amblyopone australis & 48 & 24 & $22 A+26 M$ & Austrália & 1 \\
\hline Mystrium camillae & 32 & 16 & ND & Malásia (Sarawak) & 2 \\
\hline Stigmatomma cleae & 18 & 9 & $12 M+6 A$ & Brasil-BA & NP \\
\hline Stigmatomma reclinatum & 38 & 19 & ND & Malásia & 3 \\
\hline \multicolumn{6}{|c|}{ Ectatomminae } \\
\hline Ectatomma brunneum & 44 & 22 & $22 M+22 A$ & Brasil-BA & 4 \\
\hline Ectatomma edentatum & 46 & 23 & ND & Brasil-MG & 4 \\
\hline Ectatomma muticum & - & 20 & $32 \mathrm{M}+8 \mathrm{~A}$ & Brasil-BA & 4 \\
\hline Ectatomma permagnum & 46 & 23 & $20 M+26 A$ & Brasil-BA & 4 \\
\hline Ectatomma tuberculatum & 36 & 18 & $30 \mathrm{M}+6 \mathrm{~A}$ & Brasil-BA & 4 \\
\hline Gnamptogenys annulata & 68 & 34 & $6 \mathrm{M}+62 \mathrm{~A}$ & Brasil-BA & 5 \\
\hline Gnamptogenys binghami & - & 22 & ND & Malásia & 3 \\
\hline Gnamptogenys menadensis & 42 & 21 & ND & Malásia & 3 \\
\hline Gnamptogenys moelleri & 34 & 17 & $20 M+14 A$ & Brasil-BA & NP \\
\hline Gnamptogenys pleurodon & 32 & 16 & ND & Guiana Francesa & $\mathrm{NP}$ \\
\hline Gnamptogenys sp. nv. & 46 & 23 & $16 M+30 A$ & Brasil-BA & 5 \\
\hline Gnamptogenys sp1 & 26 & 13 & $14 \mathrm{M}+12 \mathrm{~A}$ & Costa Rica & NP \\
\hline Gnamptogenys sp2 & 32 & 16 & $16 \mathrm{M}+16 \mathrm{~A}$ & Costa Rica & $\mathrm{NP}$ \\
\hline Gnamptogenys sp. 1 & 42 & 21 & ND & Malásia, Cingapura & 3,6 \\
\hline Gnamptogenys sp. 2 & 36 & 18 & ND & Malásia, Cingapura & 3,6 \\
\hline Gnamptogenys sp. 1 & 36 & 18 & ND & Brasil-BA & NP \\
\hline Gnamptogenys sp. 2 & 16 & 8 & ND & Brasil-BA & NP \\
\hline Gnamptogenys striatula & 34 & 17 & $24 M+10 A$ & Brasil-BA, MG & 5 \\
\hline Gnamptogenys triangularis & 20 & 10 & ND & Guiana Francesa & $\mathrm{NP}$ \\
\hline Rhytidoponera aciculata & 52 & 26 & $18 M+34 \mathrm{~A}$ & Austrália & 1 \\
\hline Rhytidoponera chalybaea & 42 & 21 & $6 \mathrm{M}+36 \mathrm{~A}$ & Austrália & 1 \\
\hline Rhytidoponera impressa & 42 & 21 & $6 \mathrm{M}+36 \mathrm{~A}$ & Austrália & 1 \\
\hline Rhytidoponera maniae & $44-48$ & - & Polimorfismo & Austrália & 1 \\
\hline Rhytidoponera mayri & 50 & 25 & $20 \mathrm{M}+30 \mathrm{~A}$ & Austrália & 1 \\
\hline Rhytidoponera metallica & 22 & 11 & $12 \mathrm{M}+10 \mathrm{~A}$ & Austrália & 1 \\
\hline Rhytidoponera metallica & 24 & 12 & $16 \mathrm{M}+8 \mathrm{~A}$ & Austrália & 1 \\
\hline Rhytidoponera metallica & 36 & 18 & $6 \mathrm{M}+30 \mathrm{~A}$ & Austrália & 1 \\
\hline Rhytidoponera metallica & 42 & 21 & ND & Austrália & 1 \\
\hline Rhytidoponera metallica & 46 & 23 & $4 \mathrm{M}+42 \mathrm{~A}$ & Austrália & 1 \\
\hline Rhytidoponera purpurea & 38 & 19 & $14 M+24 A$ & Austrália & 1 \\
\hline Rhytidoponera victoriae & 42 & 21 & ND & Austrália & 1 \\
\hline Typhlomyrmex meire & 20 & 10 & $12 M+8 A$ & Brasil-BA & 7 \\
\hline Typhlomyrmex rogenhoferi & 38 & 19 & $6 \mathrm{M}+32 \mathrm{~A}$ & Brasil-BA & 7 \\
\hline Typhlomyrmex rogenhoferi & 34 & 17 & $2 M+32 A$ & Brasil-PA & 7 \\
\hline Typhlomyrmex rogenhoferi & 36 & 18 & $2 M+34 A$ & Guiana Francesa & 7 \\
\hline \multicolumn{6}{|c|}{ Heteroponerinae } \\
\hline Heteroponera dollo & 24 & 12 & $22 \mathrm{M}+2 \mathrm{~A}$ & Brasil-MG & 8 \\
\hline Heteroponera relicta & 22 & 11 & $2 A+20 M$ & Austrália & 1 \\
\hline \multicolumn{6}{|c|}{ Paraponerinae } \\
\hline Paraponera clavata & 66 & 33 & $66 \mathrm{~A} ?$ & Brasil-MT & 9 \\
\hline Paraponera clavata & 54 & 27 & $12 \mathrm{M}+42 \mathrm{~A}$ & Brasil-PA & 9 \\
\hline \multicolumn{6}{|c|}{ Ponerinae } \\
\hline Anochetus altisquanis & 30 & 15 & ${ }^{*} 16 \mathrm{M}+14 \mathrm{~A}$ & Brasil-BA & 10 \\
\hline Anochetus horridus & 46 & 23 & ${ }^{*} 14 \mathrm{M}+32 \mathrm{~A}$ & Guiana Francesa & 10 \\
\hline Anochetus emarginatus & 28 & 14 & ND & Brasil-MG & $\mathrm{NP}$ \\
\hline Anochetus graeffei & 30 & 15 & ND & Indonésia & 11 \\
\hline Anochetus graeffei & 38 & 19 & ND & Índia & 12 \\
\hline
\end{tabular}




\begin{tabular}{|c|c|c|c|c|c|}
\hline Subfamília/Espécie & $2 n$ & $\mathbf{n}$ & Cariótipo diploide & Origem & FONTE \\
\hline Anochetus madaraszi & 28 & 14 & ND & Índia & 12 \\
\hline Anochetus modicus & 30 & 15 & ND & Indonésia & 11 \\
\hline Anochetus yerburyi & 30 & 15 & ND & Índia & 12 \\
\hline Anochetus sp. & 34 & 17 & ND & Sarawak & 2 \\
\hline Anochetus sp.1 & 24 & 12 & ND & Malásia, Cingapura & 6 \\
\hline Anochetus sp. 2 & & 19 & ND & Malásia, Cingapura & 6 \\
\hline Anochetus sp. 4 & 30 & 15 & ND & Índia & 12 \\
\hline Anochetus sp. 5 & 34 & 17 & ND & Índia & 12 \\
\hline Bothroponera rubiginosa & 76 & 38 & $18 M+58 A$ & Índia & 12 \\
\hline Bothroponera sp. 1 & 48 & 24 & $48 \mathrm{~A}$ & Índia & 12 \\
\hline Bothroponera sp.2 & 60 & 30 & $36 M+24 A$ & Austrália & 1 \\
\hline Bothroponera sp. 2 & 52 & 26 & $14 M+38 A$ & Índia & 12 \\
\hline Brachyponera chinensis & 22 & 11 & $20 M+2 A$ & Japão & 17 \\
\hline Brachyponera lutea & 16 & 8 & $8 M+8 A$ & Austrália & 1 \\
\hline Brachyponera luteipes & 22 & 11 & $20 M+2 A$ & Índia & 12 \\
\hline Brachyponera sp. & 22 & 11 & ND & Malásia, Cingapura & 6 \\
\hline Centromyrmex feae & 44 & 22 & ND & Índia & 12 \\
\hline Cryptopone rotundiceps & 12 & 6 & $6 M+6 A$ & Austrália & 1 \\
\hline Cryptopone sauteri & 28 & 14 & $24 A+4 M$ & Japão & 17 \\
\hline Cryptopone testacea & 18 & 9 & ND & Malásia (Sarawak) & 2,3 \\
\hline Diacamma ceylonense & 10 & 5 & ND & Índia & 22 \\
\hline Diacamma indicum & 14 & 7 & $* 10 M+4 A$ & Índia & 22 \\
\hline Diacamma 'nilgirl' & 10 & 5 & ND & Índia & 22 \\
\hline Diacamma vagans & - & 7 & ND & Índia & 12 \\
\hline Diacamma sp. & 66 & 33 & ND & Indonésia & 11 \\
\hline Diacamma sp. & 36 & 18 & ND & Malásia, Cingapura & 6 \\
\hline Diacamma sp. & 58 & 29 & ND & Malásia (Sarawak) & 2 \\
\hline Diacamma sp. 1 & 44 & 22 & ND & Malásia & 3 \\
\hline Diacama sp. 2 & 30 & 15 & ND & Índia & 12 \\
\hline Dinoponera australis & 114 & 57 & & Brasil-MG & 13 \\
\hline Dinoponera gigantea & 82 & 41 & & Brasil-PA & 13 \\
\hline Dinoponera lucida & $\begin{array}{c}106,118, \\
120 \\
\end{array}$ & 53 & $18 M+88 \mathrm{~A}$ & Brasil-BA, ES & 13 \\
\hline Dinoponera longipes & 62 & 31 & ND & Brasil-AC & NP \\
\hline Dinoponera quadriceps & 92 & 46 & ND & Brasil-BA, SE & 13 \\
\hline Ectomomyrmex astutus & 18 & 9 & ND & Indonésia & 11 \\
\hline Ectomomyrmex astutus & 22 & 11 & ND & Indonésia & 11 \\
\hline Ectomomyrmex leeuwenhoeki & 16 & 8 & ND & Malásia & 3 \\
\hline Ectomomyrmex sp. & 38 & 19 & ND & Índia & 12 \\
\hline Hypoponera confinis & 38 & 19 & ND & Indonésia & 11 \\
\hline Hypoponera pruinosa & 24 & 12 & ND & Malásia, Indonésia & 3,11 \\
\hline Hypoponera sp. & 38 & 19 & ND & Austrália & 1 \\
\hline Hypoponera sp. & 38 & 19 & ND & Austrália & 1 \\
\hline Hypoponera sp. 2 & 38 & 19 & ND & Malásia & 3 \\
\hline Hypoponera sp. 2 & 38 & 19 & $6 \mathrm{M}+32 \mathrm{~A}$ & Brasil-MG & NP \\
\hline Hypoponera sp. 2 & 38 & 19 & ND & Brasil-MG & NP \\
\hline Hypoponera sp3 & 36 & 18 & ND & Malásia & 3 \\
\hline Leptogenys borneensis & 46 & 23 & ND & Malásia & 3 \\
\hline Leptogenys diminuta & 38 & 19 & ND & Malásia, Índia & 3,12 \\
\hline Leptogenys diminuta & 32 & 16 & ND & Indonésia & 11 \\
\hline Leptogenys hysterica & 26 & 13 & ND & Índia & 12 \\
\hline Leptogenys iridescens & 46 & 23 & ND & Malásia, Indonésia & 3,11 \\
\hline
\end{tabular}




\begin{tabular}{|c|c|c|c|c|c|}
\hline Subfamília/Espécie & $2 n$ & $\mathbf{n}$ & Cariótipo diploide & Origem & FONTE \\
\hline Leptogenys kraepelini & 26 & 13 & ND & Indonésia & 11 \\
\hline Leptogenys minchini & 52 & 26 & ND & Índia & 12 \\
\hline Leptogenys myops & 48 & 24 & ND & Sarawak, Malásia, Indonésia & $2,3,11$ \\
\hline Leptogenys ocellifera & 46 & 23 & ND & Índia & 12 \\
\hline Leptogenys peuqueti & 54 & 27 & ND & Indonésia & 11 \\
\hline Leptogenys unistimulosa & 42 & 21 & ND & Brasil-BA & NP \\
\hline Leptogenys vogeli & 30 & 15 & ND & Brasil-BA & NP \\
\hline Leptogenys sp. & 54 & 27 & ND & Sarawak & 2 \\
\hline Leptogenys sp. 1 & 38 & 19 & ND & Malásia, Cingapura & 6 \\
\hline Leptogenys sp. 2 & 48 & 24 & ND & Malásia, Cingapura & 6 \\
\hline Leptogenys sp. 5 & 30 & 15 & ND & Índia & 12 \\
\hline Mayaponera constricta & 30 & 15 & $30 \mathrm{~A}$ & Brasil-BA & \\
\hline Mesoponera rubra & 20 & 10 & ND & Malásia (Sarawak) Indonésia & $2,3,11$ \\
\hline Neoponera apicalis (Ilhéus) & 36 & 18 & $28 \mathrm{M}+8 \mathrm{~A}$ & Brasil-BA & 14 \\
\hline Neoponera apicalis (Belmonte) & 40 & 20 & $30 M+10 A$ & Brasil-BA & 14 \\
\hline Neoponera apicalis (Uruçuca) & 68 & 34 & $48 M+20 A$ & Brasil-BA & 14 \\
\hline Neoponera apicalis & 38 & 19 & $16 \mathrm{M}+22 \mathrm{~A}$ & México & NP \\
\hline Neoponera apicalis & 38 & 19 & $20 M+18 A$ & Guiana Francesa & NP \\
\hline Neoponera apicalis & 68 & 34 & $6 \mathrm{M}+62 \mathrm{~A}$ & Guiana Francesa & NP \\
\hline Neoponera bactronica (Ilhéus) & 26 & 13 & $6 M+20 A$ & Brasil-BA & 14 \\
\hline Neoponera bactronica (Una) & 28 & 14 & $22 M+6 A$ & Brasil-BA & 14 \\
\hline Neoponera carinulata & 24 & 12 & $4 M+22 A$ & Brasil-BA & 14 \\
\hline Neoponera concava & 54 & & $6 \mathrm{M}+48 \mathrm{~A}$ & Brasil: BA & 14 \\
\hline Neoponera crenata & 26 & 13 & $2 M+24 A$ & Brasil-MG & 14 \\
\hline Neoponera goeldii & 24 & 12 & $24 \mathrm{~A}$ & Guiana Francesa & 14 \\
\hline Neoponera inversa & 30 & 15 & $20 M+10 \mathrm{~A}$ & Brasil-BA & 14 \\
\hline Neoponera marginata & 46 & 23 & $28 \mathrm{M}+18 \mathrm{~A}$ & Brasil-MG & 14 \\
\hline Neoponera metanotalis & 70 & 35 & $16 \mathrm{M}+54 \mathrm{~A}$ & Brasil: BA & 14 \\
\hline Neoponera moesta & 26 & 13 & $26 \mathrm{~A}$ & Brasil-MG & 14 \\
\hline Neoponera unidentata & 12 & 6 & $12 \mathrm{M}$ & Brasil-BA & 14 \\
\hline Neoponera venusta & 48 & 24 & $26 \mathrm{M}+22 \mathrm{~A}$ & Brasil-MG & 14 \\
\hline Neoponera verenae (Ilhéus) & 42 & 21 & $30 \mathrm{M}+10 \mathrm{~A}$ & Brasil-BA & 14 \\
\hline Neoponera verenae & $58-60$ & 30 & $12 \mathrm{M}+46-48 \mathrm{~A}$ & Brasil-MG & 14 \\
\hline Neoponera verenae (Ilhéus) & 62 & 31 & $14 M+48 A$ & Brasil-BA & 14 \\
\hline Neoponera verenae & 64 & 32 & $12 \mathrm{M}+52 \mathrm{~A}$ & Brasil-SP & 14 \\
\hline Neoponera verenae & 68 & 34 & $12 \mathrm{M}+56 \mathrm{~A}$ & Guiana Francesa & $\mathrm{NP}$ \\
\hline Neoponera verenae & 68 & 34 & $8 M+60 A$ & Guiana Francesa & NP \\
\hline Neoponera verenae & 60 & 30 & $12 M+48 A$ & Guiana Francesa & $\mathrm{NP}$ \\
\hline Neoponera villosa & 34 & 17 & $12 \mathrm{M}+22 \mathrm{~A}$ & Brasil-BA & 14 \\
\hline Odontomachus affinis & 44 & 22 & $* 44 \mathrm{~A}$ & Brasil-BA & 23 \\
\hline Odontomachus bauri & 44 & 22 & $* 44 \mathrm{~A}$ & Brasil-BA & 23 \\
\hline Odontomachus biumbonatus & 44 & 22 & $* 44 \mathrm{~A}$ & Brasil-BA & 23 \\
\hline Odontmachus chelifer & 44 & 22 & ${ }^{*} 4 \mathrm{M}+40 \mathrm{~A}$ & Brasil-BA & 10 \\
\hline Odontomachus haematodus & 44 & 22 & $44 \mathrm{~A}$ & Brasil-BA & 15 \\
\hline Odontomachus hastatus & 44 & 22 & ${ }^{*} 4 \mathrm{M}+40 \mathrm{~A}$ & Guiana Francesa & 16 \\
\hline Odontomachus latidens & 30 & 15 & ND & Malásia & 3 \\
\hline Odontomachus latidens & 32 & 16 & ND & Indonésia & 11 \\
\hline Odontomachus meinerti & 44 & 22 & $* 4 M+40 A$ & Brasil-BA & 10 \\
\hline Odontomachus rixosus & 30 & 15 & ND & Malásia & 3 \\
\hline Odontomachus scalptus & 44 & 22 & $44 \mathrm{~A}$ & Guiana Francesa & 16 \\
\hline Odontomachus simillimus & 44 & 22 & $44 \mathrm{~A}$ & Sarawak, Malásia, Indonésia & $2,3,11$ \\
\hline Odontomachus sp. & 44 & 22 & $44 \mathrm{~A}$ & Brasil-MG & NP \\
\hline Odontomachus sp. 1 & 44 & 22 & $22 \mathrm{~A}$ & Malásia, Cingapura & 6 \\
\hline Odontomachus sp. 1 & 44 & 22 & $4 A^{M}+40 A$ & Austrália & 1 \\
\hline Odontomachus sp. 2 & $30+1 B$ & 15 & Polimorfismo & Malásia, Cingapura & 6 \\
\hline Odontomachus sp. 3 & 44 & 22 & ND & Malásia, Cingapura & 6 \\
\hline
\end{tabular}




\begin{tabular}{|c|c|c|c|c|c|}
\hline Subfamília/Espécie & $2 n$ & $\mathbf{n}$ & Cariótipo diploide & Origem & FONTE \\
\hline Odontoponera transversa & 46 & 23 & ND & Índia & 12 \\
\hline Odontoponera transversa & 42 & 21 & ND & Indonésia & 11 \\
\hline Pachycondyla crassinoda & 62 & 31 & ND & Brasil-BA & 14 \\
\hline Pachycondyla harpax & 96 & 48 & ND & Brasil-BA & 14 \\
\hline Pachycondyla impressa & 94 & & $8 M+86 A$ & Brasil-BA & 14 \\
\hline Pachycondyla striata & 104 & 52 & ND & Brasil-BA & 14 \\
\hline Pachycondyla [sensu Brown, 1973] sp. & 28 & 14 & ND & Malásia, Cingapura & 6 \\
\hline Pachycondyla [sensu Brown, 1973] sp. & 22 & 11 & ND & Malásia, Cingapura & 6 \\
\hline Pachycondyla [sensu Brown, 1973] sp. & 36 & 18 & ND & Malásia, Cingapura & 3,6 \\
\hline Pachycondyla [sensu Brown, 1973] sp. & 44 & 22 & ND & Indonésia & 11 \\
\hline Pachycondyla [sensu Brown, 1973] sp. & $22+B$ & 11 & ND & Malásia, Cingapura & 6 \\
\hline Pachycondyla [sensu Brown, 1973] sp. & 36 & 18 & ND & Malásia & 3 \\
\hline Platy thyrea pilosula & 40 & 20 & ND & Guiana Francesa & NP \\
\hline Platy thyrea quadridenta & 18 & 9 & ND & Malásia & 3 \\
\hline Platy thyrea tricuspidata & 94 & 47 & ND & Malásia & 3 \\
\hline Ponera japonica & 12 & 6 & ND & Malásia & 3 \\
\hline Ponera scabra & 7 & 4 & $2 M+2 A$ & Japão & 17 \\
\hline Ponera pensylvanica & 12 & 6 & ND & $?$ & 18 \\
\hline Ponera sp. & 12 & 6 & $5 M+1 A$ & Japão & 19 \\
\hline Ponera sp. & 12 & 6 & ND & Indonésia & 11 \\
\hline Pseudoneoponera rufipes & 48 & 24 & $20 M+28 A$ & Índia & 12 \\
\hline Pseudoneoponera tridentata & 28 & 14 & ND & Malásia & 3 \\
\hline Pseudoponera stigma & 14 & 7 & $12 \mathrm{M}+2 \mathrm{~A}$ & Brasil-BA & 14 \\
\hline Pseudoponera gilberti & 12 & 6 & $12 \mathrm{M}$ & Brasil-BA & 14 \\
\hline Pseudoponera succedanea & 14 & 7 & $14 \mathrm{M}$ & Guiana Francesa & 14 \\
\hline Rasopone arhuaca & 36 & 18 & $36 \mathrm{~A}$ & Guiana Francesa & 14 \\
\hline Rasopone ferruginea & 38 & 19 & ${ }^{*} 16 \mathrm{M}+22 \mathrm{~A}$ & Brasil-BA & 20 \\
\hline Thaumatomyrmex contumax & 60 & 30 & $* 22 \mathrm{M}+38 \mathrm{~A}$ & Brasil-BA & NP \\
\hline Thaumatomyrmex mutilatus & 44 & 22 & ${ }^{*} 12 \mathrm{M}+32 \mathrm{~A}$ & Brasil-MG & NP \\
\hline Thaumatomyrmex mutilatus & 62 & 31 & $* 20 M+42 A$ & Brasil-BA & NP \\
\hline Thaumatomyrmex mutilatus & 34 & 17 & $* 22 M+12 A$ & Brasil-MS & NP \\
\hline Thaumatomyrmex ferox & 42 & 21 & $* 4 \mathrm{M}+38 \mathrm{~A}$ & Costa Rica & NP \\
\hline Thaumatomyrmex sp. 1 & 20 & 10 & $* 10 M+10 \mathrm{~A}$ & Brasil-BA & NP \\
\hline \multicolumn{6}{|c|}{ Proceratiinae } \\
\hline Discothyrea sp. & 30 & 15 & ND & Indonésia & 11 \\
\hline Probolomyrmex sp. & 28 & 14 & ND & Austrália & 21 \\
\hline Proceratium sp. & 48 & 24 & ND & Malásia (Sarawak) & 2 \\
\hline
\end{tabular}

$\mathrm{NP}=$ Mariano, C.S.F. Não Publicado

* = Adaptado da publicação original, nomenclatura de Levan et. al (1964)

\section{Referências relacionadas na coluna FONTE da Tabela 1:}

1 - IMAI, H. T; CROZIER R. H.; TAYLOR, R. W. Karyotype evolution in Australian ants. Chromosoma, v. 59, p. 341-393, 1977.

2 - TJAN, K.N.; IMAI, H.T.; KUBOTA, M.; BROWN Jr, W.L.; GOTWALD Jr, W.L.; YONG, H.-S.; LEH, C. Chromosome observations of Sarawak ants. Annual Report National Institute of Genetic Japan, v. 36, p. 57, 1986.

3- IMAI, H.T.; BROWN Jr, W.L.; KUBOTA, M.; YONG, H.; THO, Y.P. Chromosome observations on tropical ants from western Malaysia II. Annual Report National Institute of Genetics, Japan,v. 34, p. 66-69, 1983.
4 - BARROS, L. A. C.; MARIANO, C. S. F.; BORGES, D. S.; POMPOLO, S. G.; DELABIE, J. H. C. Cytogenetic studies of the Neotropical ant genus Ectatomma (Formicidae: Ectatomminae: Ectatommini). Sociobiology, v. 51, n. 3, p. 555-561, 2008.

5 - BORGES, D. S.; MARIANO, C. S. F.; DELABIE, J.H. C.; POMPOLO, S. G. Estudos citogenéticos em formigas neotropicais do gênero Gnamptogenys Roger (Hymenoptera, Formicidae, Ectatomminae). Revista Brasileira de Entomologia, v. 48, n. 4, p. 481-484, 2004a.

6 - GOÑI, B.; IMAI, H. T.; KUBOTA, M.; KONDO, M.; YONG, H.; THO, Y. P. Chromosome observations on tropical ants in western Malaysia and Singapore. Annual Report National Institute of Genetics, Japan, v. 32, p. 71-73, 1981. 
7 - MARIANO, C. S. F.;LACAU, S.; POMPOLO, S. G.; SPOSITO, E. C.; BORGES, D. S.; DERGAM, J. A;.VILLEMANT, C.; DELABIE,J. H. C. Cytogenetic studies in the rare neotropical ant genus Typhlomyrmex (Ectatomminae: Typhlomyrmecini). Sociobiology, v. 47, n. 1, p. 225-234, 2006a.

8 - BORGES, D. S.; DELABIE, J.H. C.; MARIANO, C. S. F.; POMPOLO, S. G. Notes écologiques et étude cytogénétique de La fourmi néotropicale Heteroponera dolo (Roger, 1861) (Hymenoptera, Formicidae, Heteroponerinae).Bulletin de la Société Entomologique de France, v. 109, n. 3, p. 257-261, 2004b.

9 - COSTA, M.S.; MARIANO, C.S.F.; FERNANDES, A. M.; POMPOLO, S. G.; DELABIE, J. H. C.Variação cariotípica em populações distantes de Paraponeraclavata(Fabricius) (Formicidae, Paraponerinae). In: XVII SIMPÓSIO DE MIRMECOLOGIA, 2005, Campo Grande. Resumos XVII Simpósio de Mirmecologia Biodiversidade e Conservação. Campo Grande: 2005, p. 1-3

10 - SANTOS, I. S.; COSTA, M. A.; MARIANO, C. S. F.; DELABIE, J. H. C.; ANDRADE-SOUZA, V.; SILVA J. G. A cytogenetic approach to the study of Neotropical Odontomachus and Anochetus ants (Hymenoptera: Formicidae). Annals of the Entomological Society of America, v. 103, n. 3, p. 424-429, 2010.

11 - IMAI, H. T.; KUBOTA, M.; BROWN JR, W. L.; IHARA, M.; TOHARI, M.; PRANATA, R. I. Chromosome observations on tropical ants from Indonesia. Annual Report National Institute of Genetics, Japan, v. 35, p. 4648, 1985.

12 - IMAI, H. T.; BARONI-URBANI, C.; KUBOTA, M.; SHARMA, G.; NARASIMHANNA, M. N.; DAS, B. C.; SHARMA, A. K.; SHARMA, A.; DEODIKAR, G. B.; VAIDYA, V. G.; RAJASEKARASETTY, M. R. Karyological survey of Indian ants. Japanese Journal of Genetics, v. 59, p. $1-32,1984 a$.

13 - SANTOS, I. S.; DELABIE, J. H. C.; SILVA J. G.; COSTA, M. A.; BARROS, L. A. C.; POMPOLO, S.G.; MARIANO, C. S. F. Karyotype differentiation among four Dinoponera (Formicidae: Ponerinae) species. Florida Entomologist, v. 95, n. 3, p. $737-742,2012$

14 - MARIANO, C. S. F.; , POMPOLO, S. G.; SILVA, J. G.; DELABIE, J. H. C. Contribution of cytogenetics to the debate on the paraphyly of Pachycondyla spp. (Hymenoptera, Formicidae, Ponerinae). Psyche, v. 2012, p. 1-9, 2012.
15 - SANTOS, I.S.; COSTA, M.A., MARIANO, C.F.S.; DELABIE, J.H.C.; SILVA, J.G. Análise citogenética em Odontomachus haematodus (Hymenoptera: Formicidae) de ocorrência no corredor central da Mata Atlântica. In: CONGRESSO BRASILEIRO DE GENÉTICA, 53, 2007, Águas de Lindoia. Resumos do $5^{\circ}$ Congresso Brasileiro de Genética, Águas de Lindoia -SP, 2007, 53.

16 - MARIANO, C. S. F.; SANTOS, I. S.; GROC, S.; LEROY, C.; MALE, P-J.; RUIZ-GONZALEZ, M. X. ; CERDAN, P.; DEJEAN, A.; DELABIE, J. H. C. The karyotypes of Gigantiops destructor (Fabricius) and other ants from French Guiana (Formicidae). Annales de la Société Entomologique de France, v. 47, n. 1, p. 140-146, 2011.

17 - IMAI, H.T.; KUBOTA, M. Karyological studies of Japanese ants (Hymenoptera, Formicidae). III. Karyotypes of nine species in Ponerinae, Formicinae, and Myrmicinae. Chromosoma, v. 37, p. 193-200, 1972.

18 - HAUSCHTECK-JUNGEN, E.; JUNGEN, H. Ant chromosomes II. Karyotypes of western paleartic species. Insectes Sociaux, v. 30, p. 149-164,1983.

19 - IMAI, H.T. Karyological studies of Japanese ants I. Chromosome evolution and species differentiation in ants. Science Report T.K.D. Sect. B., v. 206, p. 1-20, 1969.

20 - GUIMARÃES, I. N.; DELABIE, J. H. C.; FEITOSA, R. M.; MARIANO, C. S. F.. Cytogenetic characterization of Pachycondyla ferruginea (Fr. Smith,1858) and its position between the Neotropical Pachycondyla (FORMICIDAE; PONERINI). In: XXI Simpósio de Mirmecologia an international ant meeting. Fortaleza, 2013.

21 - CROZIER, R.H. The chromosomes of three Australian dacetine ant species (Hymenoptera: Formicidae). Psyche, v. 75, n. 1, p. 87-90, 1968.

22 - KARNIK et al, 2010. Karyotype instability in the ponerine ant genus Diacamma. Journal of Genetics, Vol. 89, No. 2.

23 - SANTOS, I. S.; COSTA, M. A.; MARIANO, C. S. F.; DELABIE, J. H. C.; SILVA, J. G. Cytogenetic and molecular studies of species in the genera Odontomachus and Anochetus. In: XIX Simpósio Brasileiro de Mirmecologia e I Simpósio Franco-Brasileiro de Mirmecologia, 2009, Ouro Preto - MG. Anais do XIX Simpósio Brasileiro de Mirmecologia, 2009. 


\section{Referências}

AGOSTI, D.; JOHNSON, N. F. Editors.2005. Antbase. World Wide Web electronic publication.antbase.org, version (05/2005). Acesso em 04/11/2014

BAIMAI, V. Heterochromatin accumulation and karyotypic evolution in some dipteraninsects. Zoological Studies, v. 37, n. 2, p. 75-88, 1998.

BARROS, L. A. C.; MARIANO, C. S. F.; BORGES, D. S.; POMPOLO, S. G.; DELABIE, J. H. C. Cytogenetic Studies of the Neotropical Ant Genus Ectatomma(Formicidae: Ectatomminae: Ectatommini). Sociobiology, v. 51, n. 3, p. 555-561, 2008.

BARROS, L. A. C.; MARIANO, C. S. F.; POMPOLO, S. G.; DELABIE, J. H. C. Hsc-FA and NOR bandings on chromosomes of the giant ant Dinoponera lucida Emery, 1901 (Hymenoptera: Formicidae). Comparative Cytogenetics, v. 3, n. 2, p. 97-102, 2009.

BICKFORD, D.; LOHMAN, D. J.; SODHI, N. S.; NG, P.K.L.; MEIER, R.; WINKER, K.; INGRAM, K. K.; DAS, I. Cryptic species as a window on diversity and conservation. Trends in Ecology and Evolution, vol. 22, n..3, p. 148-155, 2006.

BOGART, J. P. Evolutionary Implications of Polyploidy in Amphibians and Reptiles. Basic Life Sciences, v. 13, p. 341-378, 1980.

BOLTON, B. Synopsis and classification of Formicidae. Memoirs of the American Entomological Institute, v. 71, p. 1-370, 2003.

BOLTON, B. 2014. An online catalog of the ants of the World. Available from http://antcat.org. (accessed [13.vi.2014])

BORGES, D.S.; MARIANO, C.S.F.; DELABIE, J.H.C.; POMPOLO, S.G. Estudos citogenéticos em formigas neotropicais do gênero Gnamptogenys Roger (Hymenoptera, Formicidae, Ectatomminae). Revista Brasileira de Entomologia, v. 48, n. 4, p. 481-484, 2004 a.

BORGES, D.S.; DELABIE, J.H.C.; MARIANO, C.S.F.; POMPOLO, S.G. Notes écologiques et étude cytogénétique de la fourmi néotropicale Heteroponera dolo (Roger, 1861) (Hymenoptera, Formicidae, Heteroponerinae). Bulletin de la Société Entomologique de France, v. 109, n. 3, p. 257-261, 2004 b.

CASTAÑO-MENESES, G.; PALACIOS-VARGAS, J.G.; DELABIE, J.H.C.; MARIANO, C.S.F. Springtails (Collembola) from nests of Ponerinae (Formicidae: Ponerinae) ants in Brazilian cacao plantations. Florida Entomologist, v. 97, n. 4, p. 1864-1866, 2014.
COSTA, M.S.; MARIANO, C.S.F.; FERNANDES, A. M.; POMPOLO, S. G.; DELABIE, J.H.C. Variação cariotípica em populações distantes de Paraponera clavata (Fabricius) (Formicidae, Paraponerinae). In: XVII SIMPÓSIO DE MIRMECOLOGIA, 2005, Campo Grande. Resumos XVII SIMPÓSIO DE MIRMECOLOGIA Biodiversidade e Conservação. Campo Grande: 2005, p. 1-3.

CRISTIANO, M.P.; CARDOSO, D.C.; FERNANDESSALOMÃO, T.M. Cytogenetic and molecular analyses reveal a divergence between Acromyrmex striatus (Roger, 1863) and other congeneric species: taxonomic implications. Plos One, v. 8, n. 3, p.1-9, 2013.

CROZIER, R.H. The chromosomes of three Australian dacetine ants species (Hymenoptera: Formicidae). Psyche, v. 75, n. 1, p. 87-90,1968.

CROZIER, R.H. Animal Cytogenetics. Ed. By: B. John, H. Bauer, S. Brown, H. Kayano, A. Levan; M. White. Gebrüder Borntraeger, Berlin. 3. 94 p. 1975.

DELABIE, J.H.C.; LACAU, S.; NASCIMENTO, I.C.; CASIMIRO, A.B.; CAZORLA, I.M. Communauté des fourmis des souches d'arbres morts dans trois réserves de la forêt Atlantique brésilienne (Hymenoptera, Formicidae). Austral Ecology, v. 7, p. 95-103, 1997.

DELABIE, J.H.C.; MARIANO, C.S.F.; MENDES, L.F.; POMPOLO, S.G.; FRESNEAU, D. Problemas apontados por estudos morfológicos, ecológicos e citogenéticos no gênero Pachycondyla na Região Neotropical: o caso do complexo Apicalis. In: Insetos Sociais: da Biologia à Aplicação, E.F. Vilela, I.A. Santos, J.H. Schoereder, J.E. Serrão, L.A.O. Campos e J. Lino Neto Eds., p. 196-222. Editora da Universidade Federal de Viçosa. 2008.

FLEMMING, W. Zellsubstanz, Kern und Zelltheilung. Leipzig: F.C.W. Vogel, 1882.

FRESNEAU, D. Biologie et comportement social d'une fourmi ponérine néotropicale (Pachycondyla apicalis). Thesis Doctoral Etat. Universitd Paris-Nord, Paris, 1994.

GOKHMAN, V.E. Implication of chromosomal analysis for the taxonomy of parasitic wasps (Hymenoptera). Entomological Review, v.86, p. 1-10, 2006.

GONI, B.; IMAI, H.T.; KUBOTA, M.; KONDO, M.; YONG, H.; THO, Y.P. Chromosome observations on tropical ants in western Malaysia and Singapore. Annual Report of the National Institute of Genetics, Japan, v. 32, p. 71-73, 1981.

GOULD, S.J. Viva o brontossauro: reflexões sobre história natural. Companhia das Letras, São Paulo. 525p, 1991. 
GUERRA, M.S. Introdução à Citogenética Geral. Ed. Guanabara, Rio de Janeiro. 141p. 1988.

GUIMARÃES, I.N.; DELABIE, J.H.C.; FEITOSA, R.M.; MARIANO, C.S.F. Cytogenetic characterization of Pachycondyla ferruginea (Fr. Smith, 1858) and its position between the Neotropical Pachycondyla (Formicidae; Ponerini). In: XXI Simpósio de Mirmecologia, an International Ant Meeting, 2013, Fortaleza.

HAUSCHTECK-JUNGEN, E.; JUNGEN, H. Ant chromosomes II. Karyotypes of Western Paleartic species. Insectes Sociaux, v. 30, p. 149-164, 1983.

IMAI, H.T. Karyological studies of Japanese ants I. Chromosome evolution and species differentiation in ants. Science Reports of the Tokyo Kyoiku Daigaku Section B. v.206, p. 1-20, 1969.

IMAI, H.T. Quantitative analysis of karyotype alteration and species differentiation in mammals. Evolution, v. 37, n. 6, p. 1154-1161, 1983.

IMAI, H.T. Mutability of constitutive heterochromatin (C-bands) during eukaryotic evolution and their cytological meaning. Japanese Journal of Genetics, v. 66, p. 635-661, 1991.

IMAI, H.T.; KUBOTA, M. Karyological studies of Japanese ants (Hymenoptera, Formicidae). III. Karyotypes of nine species in Ponerinae, Formicinae, and Myrmicinae. Chromosoma, v. 37, p. 193-200, 1972.

IMAI, H.T.; MARUYAMA, T. Karyotype evolution by pericentric inversion as a stochastic process. Journal of Theoretical Biology, v.70, p. 253-261 1978.

IMAI, H.T.; CROZIER, R.H. Quantitative analysis of directionality in mammalian karyotype evolution. The American Naturalist v. 116, n. 4, p. 537-569, 1980.

IMAI, H.T; CROZIER R.H.; TAYLOR, R.W. Karyotype evolution in Australian ants. Chromosoma, v. 59, p. 341-393, 1977.

IMAI, H.T.; BROWN Jr, W.L.; KUBOTA, M.; YONG, H.; THO, Y.P. Chromosome observations on tropical ants from western Malaysia II. Annual Report National Institute of Genetics, Japan, v. 34, p. 66-69, 1983.

IMAI, H.T.; BARONI-URBANI, C.; KUBOTA, M.; SHARMA, G.; NARASIMHANNA, M.N.; DAS, B.C.; SHARMA, A.K.; SHARMA, A.; DEODIKAR, G.B.; VAIDYA, V.G.; RAJASEKARASETTY, M.R. Karyological survey of Indian ants. Japanese Journal of Genetics, v. 59, p. 1-32, 1984a.
IMAI, H.T.; KUBOTA, M.; BROWN JR, W.L.; IHARA, M.; TOHARI, M.; PRANATA, R.I. Chromosome observations on tropical ants from Indonesia. Annual Report National Institute of Genetics, Japan, v. 35, p. 46-48. 1984 b.

IMAI, H.T.; KUBOTA, M.; BROWN JR, W.L.; IHARA, M.; TOHARI, M.; PRANATA, R.I. Chromosome observations on tropical ants from Indonesia. Annual Report National Institute of Genetics, Japan, v. 35, p. 46-48, 1985.

IMAI, H. T.; MARUYAMA, T.; GOJOBOTI, T.; INOUE, Y.; CROZIER, R.H. Theoretical bases for karyotype evolution. 1. The minimum-interaction hypothesis. The American Naturalist, v.128, n. 6, p. 901-920, 1986.

IMAI, H.T.; TAYLOR, R.W.; CROSLAND, M.W.J.; CROZIER, R.H. Modes of spontaneous chromosomal mutation and karyotype evolution in ants with reference to the minimum interaction hypothesis. Japanese Journal of Genetics, v.63: p.159-185, 1988.

IMAI, H.T.; TAYLOR, R.W.; CROZIER R.H. Experimental bases for the minimum interaction theory. I. Chromosome evolution in ants of the Myrmecia pilosula species complex (Hymenoptera: Formicidae: Myrmeciinae). Japanese Journal of Genetics, v. 69, p. 137-182, 1994.

IMAI, H.T.; SATTA, Y.; TAKAHATA, N. Integrative study on chromosome evolution of mammals, ants and wasps based on the Minimum Interaction Theory. Journal of Theoretical Biology, v. 210, p. 475-497, 2001.

IMAI, H.T.; SATTA, Y.; WADA, M.; TAKAHATA, $\mathrm{N}$. Estimation of the highest chromosome number of eukaryotes based on The Minimum Interaction Theory. Journal of Theoretical Biology, v. 217, p. 6174, 2002.

KANDUL, N.P.; LUKHTANOV, V.A.; PIERCE, N.E. Karyotypic diversity and speciation in Agrodiaetus butterflies. Evolution, v. 61,p. 546-559, 2007.

KARNIK, N.; CHANNAVEERAPPA, H.; RANGANATH, H.A.; GADAGKAR, R. Karyotype instability in the ponerine ant genus Diacamma. Journal of Genetics, v. 89, n. 2, p. 173-182. 2010.

KING, M. Species evolution. The role of chromosome change. Cambridge University Press. Great Britain. 336 p. 1993.

LENHART, P.A.; DASH, S.T.; MACKAY, W.P. A revision of the giant Amazonian ants of the genus Dinoponera (Hymenoptera, Formicidae). Journal of Hymenoptera Research, v. 31, p. 119-164, 2013. 
LORITE, P.; PALOMEQUE, T. Karyotype evolution in ants (Hymenoptera: Formicidae), with a review of the known ant chromosome numbers. Myrmecological News, vol 13, p. 89-102, 2010.

LUCAS, C.; FRESNEAU, D.; KOLMER, K.; HEINZE, J.; DELABIE, J.H.C.; PHO, D.B. A multidisciplinary approach to discriminating different taxa in the complex species Pachycondyla villosa (Formicidae). Biological Journal of the Linnean Society, v. 75, p. 249-259, 2002.

LUKHTANOV, V.A.; VILA, R; KANDUL, N.P. Rearrangement of the Agrodiaetus dolus species group (Lepidoptera, Lycaenidae) using a new cytological approach and molecular data. Insects, Systematics; Evolution, v. 37, n. 3, p. 1-10, 2006.

LUKHTANOV, V.A.; DINCĂ, V.; TALAVERA, G.; VILA, R. Unprecedented within-species chromosome number cline in the Wood White Butterfly Leptidea sinapis and its significance for karyotype evolution and speciation. BMC Evolutionary Biology, v.11, n.109, p. 1-11, 2011.

MACGREGOR, H.C. An introduction to animal cytogenetics. Chapman; Hall. London, Great Britain. 238 p., 1993.

MARIANO, C.S.F.; LACAU, S.; POMPOLO, S.G.; SPOSITO, E.C.; BORGES, D.S.; DERGAM, J.A; VILLEMANT, C.; DELABIE, J.H.C. Cytogenetic studies in the rare Neotropical ant genus Typhlomyrmex (Ectatomminae: Typhlomyrmecini). Sociobiology, v. 47, n. 1, p. 225-234, 2006 a.

MARIANO, C.S.F. ; POMPOLO, S.G. ; LACAU, S.; DELABIE, J.H.C. Questions sur la monophylie du taxon Pachycondyla Smith, 1858: approche cytogénétique sur le sous-genre Pachycondyla sensu Emery, 1901 (Hymenoptera: Formicidae: Ponerinae). Bulletin de la Société Entomologique de France, v. 111, p. 299-304. 2006b.

MARIANO, C.S.F.; POMPOLO, S.G.; BARROS, L.A.C.; MARIANO-NETO, E.; CAMPIOLO, S.; DELABIE, J.H.C. A biogeographical study of the threatened ant Dinoponera lucida Emery (Hymenoptera: Formicidae: Ponerinae) using a cytogenetic approach. Insect Conservation and Diversity, v. 1, p. 161-168. 2008.

MARIANO, C.S.F.; SANTOS, I.S.; GROC, S.; LEROY, C.; MALE, P-J.; RUIZ-GONZALEZ, M.X. ; CERDAN, P.; DEJEAN, A.; DELABIE, J.H.C. The karyotypes of Gigantiops destructor (Fabricius) and other ants from French Guiana (Formicidae). Annales de la Société Entomologique de France, v. 47, n. 1, p. 140-146, 2011.
MARIANO, C.S.F.; POMPOLO, S.G.; SILVA, J. G.; DELABIE, J.H.C. Contribution of cytogenetics to the debate on the paraphyly of Pachycondyla spp. (Hymenoptera, Formicidae, Ponerinae). Psyche, v. 2012, p. 1-9, 2012.

MAYR, E. Wu's genic view of speciation. Journal of Evolutionary Biology, vol. 14, p. 866-867, 2001.

MENEZES, R.S.T.; CARVALHO, A.F.; CORREIA, J.P.S.O.; SILVA, T.S.; SOMAVILLA, A.; COSTA, M.A. Evolutionary trends in the chromosome numbers of swarm-founding social wasps. Insectes Sociaux, v.61, p. 385-393, 2014.

METZ, C.H. 1916. Chromosome studies on the Diptera II. The paired association of chromosomes in the Diptera, and its significance. Journal of Experimental Zoology, 21(2): 213-279.

MOREAU, C.S.; BELL, C.D.; VILA, R.; ARCHIBALD, B.; PIERCE, N.E. Phylogeny of the ants: diversification in the age of angiosperms. Science, v. 312, p. 101-104, 2006.

OTTO, S.P.; WHITTON, J. Poliploidy incidence and evolution. Annual Review of Genetics, v. 34, p. 401437,2000 .

PADIAL, M.; RIVA, T.A. Response to recent proposals for integrative taxonomy. Biological Journal of the Linnean Society, vol. 101, p. 747-756, 2010.

SANTOS, I.S.; COSTA, M.A., MARIANO, C.F.S.; DELABIE, J.H.C.; SILVA, J.G. Análise citogenética em Odontomachus haematodus (Hymenoptera: Formicidae) de ocorrência no corredor central da Mata Atlântica. In: Resumos do $5^{\circ}$ Congresso Brasileiro de Genética, Águas de Lindoia -SP, 2007, 53.

SANTOS, I.S.; COSTA, M.A.; MARIANO, C.S.F.; DELABIE, J.H.C.; SILVA, J.G. Cytogenetic and molecular studies of species in the genera Odontomachus and Anochetus. In: Anais do XIX Simpósio Brasileiro de Mirmecologia, Ouro Preto - MG, 2009.

SANTOS, I.S.; COSTA, M.A.; MARIANO, C.S.F.; DELABIE, J.H.C.; ANDRADE-SOUZA, V.; SILVA J.G.A. Cytogenetic approach to the study of Neotropical Odontomachus and Anochetus ants (Hymenoptera: Formicidae). Annals of the Entomological Society of America, v. 103, n. 3, p. 424-429, 2010.

SANTOS, I. S.; DELABIE, J. H. C.; SILVA J. G.; COSTA, M. A.; BARROS, L. A. C.; POMPOLO, S.G.; MARIANO, C.S.F. Karyotype differentiation among four Dinoponera (Formicidae: Ponerinae) species. Florida Entomologist, v. 95, n. 3, p. 737-742, 2012. 
SBILORDO, S.H.; MARTIN, O.Y.; WARD, P. I. The karyotype of the yellow dung fly, Scathophaga stercoraria, a model organism in studies of sexual selection. Journal of Insect Science, v. 10, p. 1-11, 2010.

SCHLICK-STEINER, B.C.; STEINER, F.M.; SEIFERT, B.; STAUFFER, C.; CHRISTIAN, E.; CROZIER, R.H. Integrative taxonomy: a multisource approach to exploring biodiversity. Annual Review of Entomology, v. 55, p. 421-438, 2010.

SCHMIDT, C.A.; SHATTUCK, S.O. The Higher Classification of the Ant Subfamily Ponerinae (Hymenoptera: Formicidae), with a Review of Ponerine Ecology and Behavior. $1^{\text {st }}$ ed., Auckland: Magnolia Press, 242 p., 2014.

SEIFERT, B. Cryptic species in ants (Hymenoptera: Formicidae) revisited: we need a change in the alpha taxonomic approach. Myrmecological News, v. 12, p. 149-166. 2009.

SPIRITO, F. The role of chromosomal change in speciation. In: Endless forms. Species and speciation. Ed. by: D.J. Howard and S.H. Berlocher. Oxford University Press. New York, USA. 470 p. 1998.
SUMNER, A.T. Chromosomes: Organization and Function. Blackwell Publishing, Oxford, UK. 2003.

TJAN, K.N.; IMAI, H.T.; KUBOTA, M.; BROWN Jr, W.L.; GOTWALD Jr, W.L.; YONG, H.-S.; LEH, C. Chromosome observations of Sarawak ants. Annual Report of the National Institute of Genetics, Japan, v. 36, p. 57, 1986.

WARD, P.S. Integrating molecular phylogenetical results into ant taxonomy (Hymenoptera: Formicidae)". Myrmecological News, v. 15, p. 21-29, 2011.

WARD, P.S. The phylogeny and evolution of ants. Annual Review of Ecology, Evolution and Systematics, v. 15, n. 10, p. 1-21, 2014.

WHITE, M.J.D. Animal Cytology and Evolution. Cambridge University Press. Cambridge. 961 p. 1973.

WILD, A. Taxonomic revision of the Pachycondyla apicalis species complex (Hymenoptera: Formicidae). Zootaxa, v. 834, p. 1-25, 2005. 